\title{
PAPER
}

\section{Geometrical and topological study of the Kosterlitz-Thouless phase transition in the $X Y$ model in two dimensions}

To cite this article: Ghofrane Bel-Hadj-Aissa et al J. Stat. Mech. (2021) 023206

View the article online for updates and enhancements.

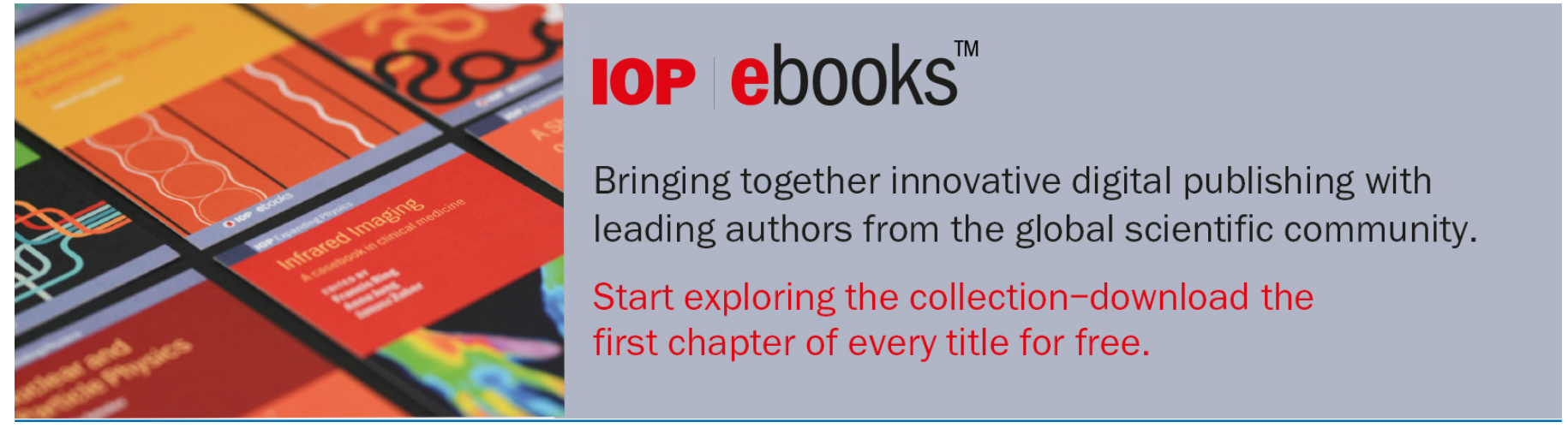

This content was downloaded from IP address 193.205.148.189 on 24/02/2021 at 21:24 


\title{
Geometrical and topological study of the Kosterlitz-Thouless phase transition in the $X Y$ model in two dimensions
}

\author{
Ghofrane Bel-Hadj-Aissa ${ }^{1,2}$, Matteo Gori ${ }^{3}$, \\ Roberto Franzosi ${ }^{2}$ and Marco Pettini ${ }^{4, *}$ \\ ${ }^{1}$ Dipartimento di Scienze Fisiche, della Terra e dell'Ambiente (DSFTA), \\ University of Siena, Via Roma 56, 53100 Siena, Italy \\ ${ }^{2}$ QSTAR \& CNR - Istituto Nazionale di Ottica, Largo Enrico Fermi 2, \\ I-50125 Firenze, Italy \\ ${ }^{3}$ Quantum Biology Lab, Howard University, 2400 6th St NW, Washington, \\ DC 20059, United States of America \\ ${ }^{4}$ Aix-Marseille University, Marseille, France, CNRS Centre de Physique \\ Théorique UMR7332, 13288 Marseille, France \\ E-mail: ghofrane.belhadjaissa@gmail.com, gori6matteo@gmail.com, \\ roberto.franzosi@ino.it and pettini@cpt.univ-mrs.fr
}

Received 19 August 2020

Accepted for publication 9 December 2020

Published 24 February 2021

Online at stacks.iop.org/JSTAT/2021/023206

https://doi.org/10.1088/1742-5468/abda27

\begin{abstract}
Phase transitions do not necessarily correspond to a symmetrybreaking phenomenon. This is the case of the Kosterlitz-Thouless (KT) phase transition in a two-dimensional classical $X Y$ model, a typical example of a transition stemming from a deeper phenomenon than a symmetry-breaking. Actually, the KT transition is a paradigmatic example of the successful application of topological concepts to the study of phase transition phenomena in the absence of an order parameter. Topology conceptually enters through the meaning of defects in real space. In the present work, the same kind of KT phase transition in a two-dimensional classical $X Y$ model is tackled by resorting again to a topological viewpoint, however focussed on the energy level sets in phase space rather than on topological defects in real space. Also from this point of view, the origin of the
\end{abstract}

*Author to whom any correspondence should be addressed. 
Geometrical and topological study of the Kosterlitz-Thouless phase transition in the $\mathbf{X Y}$ model in two dimensions

KT transition can be attributed to a topological phenomenon. In fact, the transition is detected through peculiar geometrical changes of the energy level sets which, after a theorem in differential topology, are direct probes of topological changes of these level sets.

Keywords: classical phase transitions, dynamical processes, numerical simulations

\section{Contents}

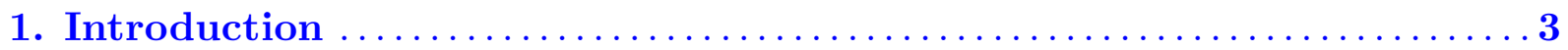

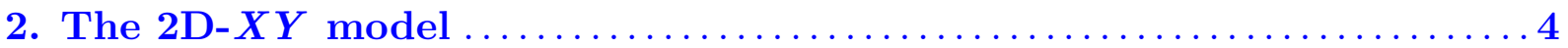

3. A bridge between topology and extrinsic geometry of energy surfaces of the phase space $\ldots \ldots \ldots \ldots \ldots \ldots \ldots \ldots \ldots \ldots \ldots \ldots \ldots \ldots \ldots \ldots \ldots \ldots \ldots \ldots \ldots \ldots$

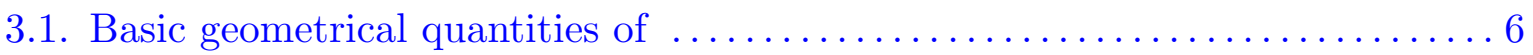

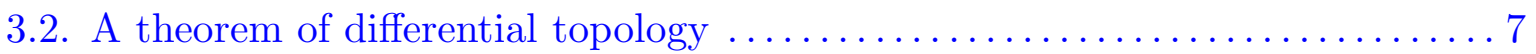

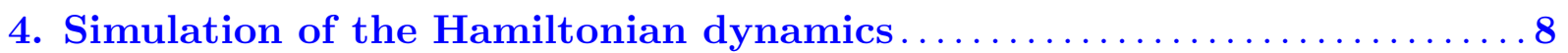

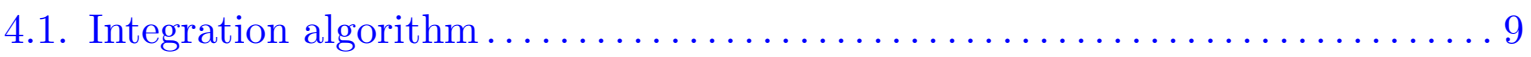

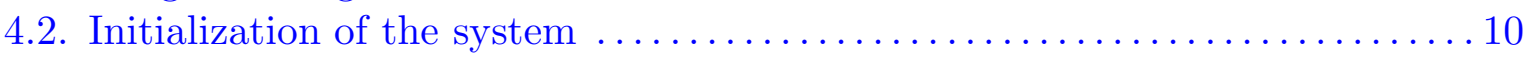

4.3. Numerical evidence of the KT phase transition .................. 10

5. Numerical results on the geometry and the topology of the subman-

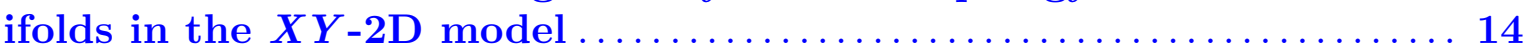

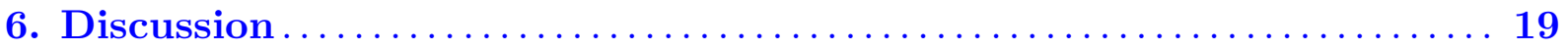

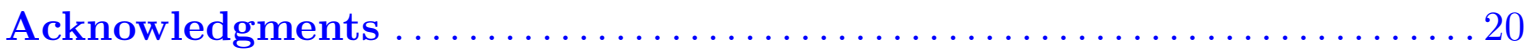

$\begin{array}{ll}\text { Appendix } & \mathbf{2 0}\end{array}$

A.1. Review of differential extrinsic geometry of codimension submanifolds ......20

A.2. Differential geometrical structure of co-dimension two submanifold in

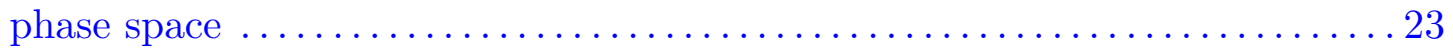

A.3. Federer-Lawrence formula for co-dimension two manifold and microcanon-

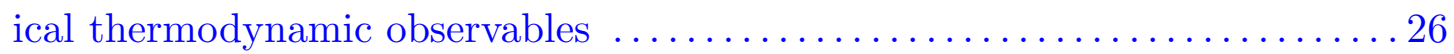

A.4. Geometrical and topological observables in $X Y$ 2D model $\ldots \ldots \ldots \ldots \ldots . \ldots 28$

$\begin{array}{ll}\text { References } & 31\end{array}$ 
Geometrical and topological study of the Kosterlitz-Thouless phase transition in the $\mathbf{X Y}$ model in two dimensions

\section{Introduction}

Phase transitions (PTs) encompass a very large number of phenomena that span from the cosmological scale down to the subnuclear scale and thus cover a huge range of energies and spatial dimensions. Despite their relevance and the extremely vast literature on this topic, a general and complete theory is not yet available. The successful and powerful phenomenological theory due to Landau associates PT with the spontaneous symmetry breaking phenomenon. However, there are many systems undergoing PT in the absence of symmetry-breaking: the liquid-gas PT which is a first order transition; systems with continuous symmetry which cannot be broken spontaneously at any finite temperature in dimensions two or lower according to the Mermin-Wagner theorem [1]; systems with local symmetries (gauge theories) which, after the Elitzur theorem [2], undergo PTs in the absence of an order parameter; glasses and supercooled liquids; amorphous and disordered systems; homopolymers and proteins undergoing folding transitions.

A paradigmatic example of $\mathrm{PT}$ in the absence of symmetry-breaking is provided by the Kosterlitz-Thouless (KT) transitions [3] which manifest in several kinds of twodimensional systems in condensed matter, such as the $X Y$ ferromagnet [4] describing spins on a 2D lattice, or a $2 \mathrm{D}$ Bose liquid as in the case of a $2 \mathrm{D}$ film of superfluid ${ }^{4} \mathrm{He}$, with $O(2)$ symmetry [5], or two-dimensional superconductors [6], or a 2D liquid crystal [7], 2D melting into a 'liquid crystal' phase with sixfold orientational order [8], or the 'quasi-condensation' of a uniform 2D fluid of identical bosons [9].

Other transitional phenomena which are experimentally well known but are still at odds with the existing theoretical frameworks, are PTs occurring in small systems, far from the thermodynamic limit which is commonly considered as a necessary requisite after the Yang-Lee theory [10]. Among many examples we can mention the formation of nanoscopic snowflakes [11], Bose-Einstein condensation [12], homopolymer [13] and protein [14] folding. Therefore the motivation for a better understanding of the deep origin of PTs is mainly twofold: there are many PT phenomena occurring without a symmetry-breaking that are obviously not encompassed by the Landau-Ginzburg theory, and there are PTs occurring in nanoscopic or mesoscopic systems (far from the thermodynamic limit) that are not encompassed by the Yang-Lee theory or by the Dobrushin-Ruelle theory [15].

During the last two decades, the mentioned deeper level of description of PTs has been found to be rooted in a novel and successful explanation of the origin of chaos in Hamiltonian dynamics tackled by means of Riemannian differential geometry [16]. Actually, a Hamiltonian flow can be identified with a geodesic flow on a Riemannian manifold equipped with a suitable metric tensor. Then, by combining together this geometrization of Hamiltonian dynamics with the microcanonical study of PT by means of Hamiltonian dynamics, a question arose naturally: are there peculiar changes of the geometry of the mechanical manifolds in correspondence of a phase transition? The answer turned out in the affirmative and, more precisely, it has been found that the topological properties of certain submanifolds of phase space are at the very grounds of the occurrence of PT [16]. For a system described by a Hamiltonian of the form $H(p, q)=\sum_{i=1}^{N} \frac{1}{2} p_{i}^{2}+V\left(q_{1}, \ldots, q_{N}\right)$, the relevant manifolds are equivalently the energy level sets $\Sigma_{E}=\left\{H\left(p_{1}, \ldots p_{N}, q_{1}, \ldots, q_{N}\right)=E \in \mathbb{R}\right\}$ and the balls 
Geometrical and topological study of the Kosterlitz-Thouless phase transition in the $\mathbf{X Y}$ model in two dimensions

$\left\{M_{E}=H^{-1}((-\infty, E])\right\}_{E \in \mathbb{R}}$ bounded by the $\Sigma_{E}$ in phase space, or the potential level sets $\Sigma_{v}=\left\{V\left(q_{1}, \ldots, q_{N}\right)=v \in \mathbb{R}\right\}$ and the balls $\left\{M_{v}=V^{-1}((-\infty, v])\right\}_{v \in \mathbb{R}}$ bounded by the $\Sigma_{v}$ in configuration space.

The main difference between the topological approach and the usual association of PTs with some kind of singularity of the statistical measures is that the topological approach puts in evidence that all the information concerning the appearance of a PT is already 'encoded' in the interactions among the degrees of freedom of a system. A fact which is upstream of any signature, singular or not, of a PT. Otherwise said, the statistical measures are conceptual tools, not directly accessible to experiments, the singularities of which are used to interpret the occurrence of PTs phenomena, to the contrary the interactions among the constituents of a system have their own physical reality (experimentally accessible) and their knowledge is sufficient to predict the occurrence of a PT by suitably analysing the energy level sets $H\left(p_{1}, \ldots, p_{N}, q_{1}, \ldots, q_{N}\right)=E$, or the potential energy level sets $V\left(q_{1}, \ldots, q_{N}\right)=v$.

Besides its application to more traditional transitions [17], this topological approach has been already successfully used to tackle the PT of a gauge model in the absence of an order parameter [18]. Moreover, peculiar topological changes entailing a PT can take place at any finite number of degrees of freedom, thus allowing to go beyond the thermodynamic limit dogma.

Remarkably, topological concepts are at the core of the KT theory independently of the above mentioned approach, in fact for the planar model of classical spins and for a superfluid two dimensional film there are spatial vortexes and their cores are holes in the surface where the system lives. Hence a vortex is called a topological defect [20]. Adding holes to a surface makes a change of its homotopy type.

Also in the present work we aim at characterizing the KT phase transition of the $X Y$-2D model in a topological framework, however, instead of looking at topological changes in real space we will focus on certain aspects of the geometry - tightly related to the topology — of the high dimensional manifolds $\Sigma_{E}$ and $M_{E}$ in phase space.

A previous attempt to tackle the topological origin of the KT transition by suitably sampling the critical points of the potential function is reported in [19] where the authors checked a conjecture about a possible origin of a singularity in configurational entropy density. Even though the conjecture put forward had reasonable basis, its application to the KT transition turned out to be inconclusive, as recognized by the authors, perhaps because of numerical limitations of the hard task of finding an adequate set of critical points of the potential.

\section{The $2 \mathrm{D}-\mathrm{XY}$ model}

The $X Y$ model is a two-vector model on a two-dimensional lattice $\mathbb{Z}^{2}$ : a two-dimensional unit-vector $\mathbf{s}_{i}=\left(s_{i x}, s_{i y}\right)=\left(\cos \theta_{i}, \sin \theta_{i}\right)$ is associated to each site $i \in \lambda^{2}$.

The standard Hamiltonian for this model is

$$
H_{\mathrm{std}}(\theta)=-J \sum_{\langle i, j\rangle=1}^{N} \mathbf{s}_{i} \cdot \mathbf{s}_{j}=-J \sum_{\langle i, j\rangle \in \lambda^{2}} \cos \left(\theta_{i}-\theta_{j}\right)
$$


Geometrical and topological study of the Kosterlitz-Thouless phase transition in the $\mathbf{X Y}$ model in two dimensions

where $\langle i, j\rangle$ indicates all the pairs of nearest neighbors (resulting in short-range interactions) and $J$ is the coupling constant. Such a model can be regarded as a generalization of the Ising model in the sense that spins can rotate in two dimensions instead of pointing only in two directions. Moreover, the Hamiltonian is invariant under the action of the symmetry group $O(2)$, so that Kosterlitz and Thouless proved that the Mermin-Wagner theorem applies here because this continuous symmetry is not spontaneously broken at finite temperature, but there is still a phase transition, with a diverging correlation length, at some finite temperature in this system. Below some critical temperatures, the two-point spin correlation $\langle\mathbf{s}(r) \cdot \mathbf{s}(0)\rangle=(r / a)^{-k_{\mathrm{B}} T / 2 \pi J}$, where $a$ is the lattice spacing, is asymptotically vanishing for $r \rightarrow \infty$, so that there is no long ranged order, whereas approaching the transition temperature from above it is $\langle\mathbf{s}(r) \cdot \mathbf{s}(0)\rangle=\mathrm{e}^{-r / \xi}$, where $\xi$ is the spatial correlation length.

In this work we tackle a modified version of the $X Y$-model in equation (1) by adding a standard quadratic kinetic energy term of the conjugate momenta $p_{i}$, together with a fixed constant $2 N J$ so that the low energy limit of the potential function is well approximated by the potential energy of a set of coupled harmonic oscillators. This modified Hamiltonian reads as

$$
H(\boldsymbol{p}, \boldsymbol{\theta})=\sum_{i=1}^{n} \sum_{j=1}^{n} \frac{p_{(i, j)}^{2}}{2}+J\left[\cos \left(\theta_{(i, j)}-\theta_{(i, j+1)}\right)+\cos \left(\theta_{(i, j)}-\theta_{(i+1, j)}\right)-2\right] .
$$

which is considered with periodic boundary conditions. According to Noether's theorem, this system has two first integrals, the total energy $H(\boldsymbol{p}, \boldsymbol{\theta})=E$ and the total momen-

tum $P(\boldsymbol{p})=\sum_{i \in \lambda} p_{i}$ associated to the global $O(2)$ symmetry $\theta_{i} \rightarrow \theta_{i}+\alpha$. Thus the flow associated with the Hamiltonian (2) belongs to the regular submanifolds $\Sigma_{E, \mathscr{P}}$ of phase space $\Lambda$, that is $\Sigma_{E, \mathscr{P}}=\{x \in \Lambda \mid H(x)=E \wedge P(x)=\mathscr{P}\}$. We have $N=\operatorname{dim} \Lambda=$ $2(n \times n)$.

The only way of tackling the KT transition on the basis of the Hamiltonian in equation (1) is through the canonical statistical ensemble, whereas by rewriting the model Hamiltonian as in equation (2) allows us to tackle the same problem in the microcanonical statistical ensemble because the invariant measure of the phase space flow associated to this nonintegrable Hamiltonian is the microcanonical measure.

\section{A bridge between topology and extrinsic geometry of energy surfaces $\Sigma_{E, \mathscr{P}}$ of the phase space}

According to the topological hypothesis on the origin of PT mentioned in the Introduction, a phase transition stems from a suitable topological change of the energy level sets, immersed into the phase space of a given system. Therefore, one is confronted with problem of computing topological invariants of the regular $m$-dimensional manifolds $\Sigma_{E, f}^{m}$, level sets of energy and other first integrals of motions. This problem is, in general, a tough task, as the cohomology groups of a manifold can be directly computed only in very few cases through algebraic topology techniques. However, in some 


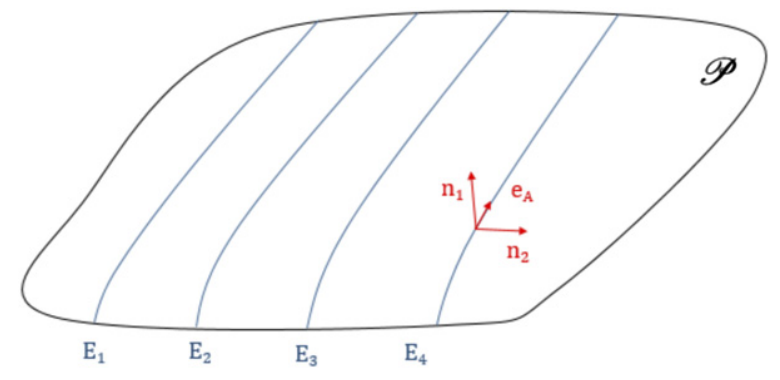

Figure 1. Level sets $H=E$ over the submanifolds $P=\mathscr{P}$ immersed into the phase space $\Lambda$.

cases it is possible to compute or, at least, to estimate topological invariants of submanifolds using the few existing results that make a link between global geometry and topology.

\subsection{Basic geometrical quantities of $\Sigma_{E, \mathscr{P}}$}

We are interested in studying the extrinsic geometry of the codimension-two submanifolds of phase space $\Sigma_{E, \mathscr{P}}$ when $\mathscr{P}$ is fixed while $E$ can change. So, we simplify the problem by considering $\Sigma_{E, \mathscr{P}}$ as a codimension-one submanifold, immersed into $\Sigma_{\mathscr{P}}$, foliated by the level sets $H=E$.

This is schematically represented in figure 1 where $n_{1}$ and $n_{2}$ are normal vector fields to $\Sigma_{E, \mathscr{P}}$, and the vector field $e_{A}$ belongs to the tangent bundle of $\Sigma_{E, \mathscr{P}}$, i.e. $e_{A} \in T \Sigma_{E, \mathscr{P}}$, (see appendices A.1 and A.2). The vector $n_{2}$ is defined through the function

$$
f_{\mathrm{HP}}=H-\frac{1}{N} \frac{P^{2}}{2} \text {. }
$$

as

$$
n_{2}=\frac{\nabla f_{\mathrm{HP}}}{\left\|\nabla f_{\mathrm{HP}}\right\|}
$$

which is necessary to define relevant geometric quantities.

A basic geometric quantity characterizing the extrinsic geometry of $\Sigma_{E, \mathscr{P}}$ is the mean curvature $M_{n_{2}}$ along the vector $n_{2}$ given by (see appendix A.4)

$$
M_{n_{2}}=\frac{1}{2 N-2}\left[\frac{\operatorname{Hess} f_{\mathrm{HP}}\left(\nabla f_{\mathrm{HP}}, \nabla f_{\mathrm{HP}}\right)}{\left\|\nabla f_{\mathrm{HP}}\right\|^{3}}-\frac{\Delta f_{\mathrm{HP}}}{\left\|\nabla f_{\mathrm{HP}}\right\|}\right] .
$$

To get some meaningful information about the topology of the co-dimension-one manifolds $\left\{\Sigma_{E, \mathscr{P}}\right\}$ immersed in the Euclidean space $\Sigma_{\mathscr{P}}$, the dispersion of the principal curvatures $\sigma_{n_{2}}^{2}\left(k_{i}\right)$ is computed from

$$
\sigma_{n 2}^{2}\left(k_{i}\right)=\left\langle k_{i}^{2}\right\rangle-\left\langle k_{i}\right\rangle^{2}=\frac{\operatorname{Tr}\left(\mathscr{W}_{n_{2}}^{2}\right)}{2 N-2}-\frac{\left(\operatorname{Tr} \mathscr{W}_{n_{2}}\right)^{2}}{(2 N-2)^{2}},
$$

where $\mathscr{W}$ is the shape-operator (Weingarten map) defined in equation (37) of the appendix A.1, where $\operatorname{Tr} \mathscr{W}_{n_{2}} /(2 N-2)=M_{n_{2}}$, and where 
Geometrical and topological study of the Kosterlitz-Thouless phase transition in the $\mathbf{X Y}$ model in two dimensions

$$
\begin{aligned}
\operatorname{Tr}\left(\mathscr{W}_{n_{2}}^{2}\right)= & \operatorname{Tr}\left[\frac{\left(\operatorname{Hess} f_{\mathrm{HP}}\right)^{2}}{\left\|\nabla f_{\mathrm{HP}}\right\|^{2}}\right]-\frac{\left(\operatorname{Hess} f_{\mathrm{HP}} \nabla f_{\mathrm{HP}}\right)^{2}}{\left\|\nabla f_{\mathrm{HP}}\right\|^{4}}+ \\
& -\frac{1}{\left\|\nabla f_{\mathrm{HP}}\right\|^{2}}\left[\frac{\left(\| \operatorname{Hess} f_{\mathrm{HP}}\right)\left(\nabla f_{\mathrm{HP}}\right) \|^{2}}{\left\|\nabla f_{\mathrm{HP}}\right\|^{2}}-\frac{\left\|\left(\operatorname{Hess} f_{\mathrm{HP}}\right)\left(\nabla f_{\mathrm{HP}}\right)^{2}\right\|^{2}}{\left\|\nabla f_{\mathrm{HP}}\right\|^{4}}\right] .
\end{aligned}
$$

An important quantity connecting thermodynamics to the differential geometry of $\Sigma_{E, \mathscr{P}}$ is the geometrical temperature given by

$$
T_{\text {geo }}(E, \mathscr{P})=\left(\int_{\Sigma_{E, \mathscr{P}}} \mu \operatorname{div} \xi\right)^{-1}=\left(\langle\operatorname{div} \xi\rangle_{\mu c}(E, \mathscr{P})\right)^{-1}
$$

where $\xi=\frac{n_{2}}{\left\|\nabla f_{\mathrm{HP}}\right\|}$ and $\langle\cdot\rangle_{\mu c}(E, \mathscr{P})$ indicates the averages over the energy level sets $\Sigma_{E, \mathscr{P}}$ with the probability measure $\mu$.

(for all the details of this section, see all of the appendices).

\subsection{A theorem of differential topology}

One of the mentioned possibilities of relating geometrical and topological properties of a manifold is provided by Pinkall's theorem [21]. In order to apply it we first need to define the dispersion of the principal curvatures $\left\{k_{i}\right\}_{i=1, \ldots, n-m}$, for any submanifold $\mathscr{M}^{m}$ of codimension $m$ immersed in an Euclidean space $\mathbb{E}^{n}$ (for details, see appendix A.2), as follows

$$
\sigma^{2}\left(k_{i}\right)=\frac{1}{(n-m)^{2}} \sum_{i<j}\left(k_{i}-k_{j}\right)^{2}=\frac{1}{n-m} \sum_{i=1}^{n-m}\left(k_{i}-\bar{k}\right)^{2}, \quad \bar{k}=\frac{1}{n-m} \sum_{i=1}^{n-m} k_{i} .
$$

Then the Pinkall's inequality reads

$$
\frac{1}{\operatorname{Vol}\left(\mathbb{S}^{n}\right)} \int_{\mathrm{N} \mathscr{M}^{m}}\left[\sigma_{\xi}^{2}\left(k_{i}\right)\right]^{(n-m) / 2} \mathrm{~d} \xi \geqslant \sum_{i=1}^{n-m-1}\left(\frac{i}{n-m-i}\right)^{(n-m) / 2-i} b_{i}\left(\mathscr{M}^{m}\right),
$$

where $\operatorname{Vol}\left(\mathbb{S}^{n}\right)$ is the volume of the $n$-dimensional sphere of unit radius, $\mathrm{N} \mathscr{M}^{m}$ is the unitary normal bundle over $\mathscr{M}^{m}$, d $\xi$ is the volume element on it and $b_{i}\left(\mathscr{M}^{m}\right)$ is the $i$ th Betti number (the diffeomorphism-invariant dimension of the $i$ th cohomology groups of the submanifold $\mathscr{M}^{m}$ ) [22]. We notice that on the right-hand side of equation (10) there is a weighted sum of Betti numbers: the weights $w_{i}=[i /(n-i)]^{n / 2-i}$ emphasize the contributions coming from the (co)homology groups $H_{i}\left(\mathscr{M}^{m}\right)$ with $i \simeq n / 2$.

In [18], Pinkall's theorem was applied to the case of a system undergoing a thermodynamic phase transition in the absence of a global symmetry-breaking, where it 
Geometrical and topological study of the Kosterlitz-Thouless phase transition in the $\mathbf{X Y}$ model in two dimensions

has been shown that the phase transition is marked by an abrupt change in both the geometry and the topology of the potential level sets $\Sigma_{V}$. A direct numerical computation of the geometrical quantity in the left-hand side of equation (10) is prohibitive for large Hamiltonian systems because for large values of $n(n \gtrsim 100)$, the $(n-m) / 2$-th power of $\sigma_{\xi}^{2}\left(k_{i}\right)$ is practically untreatable. This difficulty can be overcome by resorting to Hölder inequality [23], that allows to consider the $2 /(n-m)$-th power of the integral in equation (10) for a codimension-one manifold $\Sigma \in \mathbb{R}^{N}$

$$
\int_{\Sigma}\left[\sigma\left(k_{i}\right)\right]^{2} \mathrm{~d} \mu \leqslant\left[\int_{\Sigma}\left\{\left[\sigma\left(k_{i}\right)\right]^{2}\right\}^{(n-1) / 2} \mathrm{~d} \mu\right]^{2 /(n-1)}\left[\int_{\Sigma} \mathrm{d} \mu\right]^{1 /[1-2 /(n-1)]},
$$

that for large $n$ becomes

$$
\left[\int_{\Sigma} \mathrm{d} \mu\right]^{-1} \int_{\Sigma}\left[\sigma\left(k_{i}\right)\right]^{2} \mathrm{~d} \mu \leqslant\left[\int_{\Sigma}\left\{\left[\sigma\left(k_{i}\right)\right]^{2}\right\}^{n / 2} \mathrm{~d} \mu\right]^{2 / n} .
$$

Moreover, Hölder inequality becomes an equality when $\left[\sigma\left(k_{i}\right)\right]^{n}$ equals its average value almost everywhere on $\Sigma$. So we can introduce a reminder $r(\Sigma)$ to rewrite the last equation as

$$
\left[\int_{\Sigma} \mathrm{d} \mu\right]^{-1} \int_{\Sigma}\left[\sigma\left(k_{i}\right)\right]^{2} \mathrm{~d} \mu=\left[\int_{\Sigma}\left\{\left[\sigma\left(k_{i}\right)\right]^{2}\right\}^{n / 2} \mathrm{~d} \mu\right]^{2 / n}-r(\Sigma)
$$

where we recognize on the left-hand side the average with respect to the measure $\mathrm{d} \mu$ of the dispersion of principal curvatures. In the case where the dispersion of the principal curvatures of $\Sigma$ displays a limited variability from point to point, the remainder $r(\Sigma)$ turns to be a small correction and, consequently, the Hölder inequality is tight

$$
\begin{aligned}
\left\langle\left[\sigma\left(k_{i}\right)\right]^{2}\right\rangle_{\mu} & =\left[\int_{\Sigma}\left\{\left[\sigma\left(k_{i}\right)\right]^{2}\right\}^{n / 2} \mathrm{~d} \mu\right]^{2 / n}-r(\Sigma) \\
& =\left[\operatorname{Vol}\left(\mathbb{S}^{n}\right) \sum_{i=1}^{n}\left(\frac{i}{n-i}\right)^{n / 2-i} b_{i}(\Sigma)\right]^{2 / n}-r(\Sigma)
\end{aligned}
$$

under the hypothesis that $\mathrm{d} \mu$ is the induced Riemannian measure on $\Sigma$.

\section{Simulation of the Hamiltonian dynamics}

The standard numerical approach to the study of the KT transition has been through Monte Carlo canonical algorithms. An alternative approach, that is, performed in the framework of microcanonical ensemble, has been put forward two decades ago in reference [24], where the transitional behaviour of 2D XY model-described by the Hamiltonian in equation (2) - was first investigated by means of the associated Hamiltonian flow for which the ergodic invariant measure is the microcanonical measure. In what follows this approach is adopted to investigate the underlying geometrical/topological origin of the KT transition. It is worth mentioning that sophisticated microcanonical Monte Carlo algorithms are available [25, 26] and, in principle, these could be used 
Geometrical and topological study of the Kosterlitz-Thouless phase transition in the $\mathbf{X Y}$ model in two dimensions

as well, however Hamiltonian dynamics has the advantage of naturally sampling a constant energy surface with the appropriate measure, and it allows to compute dynamical observables, like Lyapunov exponents, that are not accessible via Monte Carlo simulations. Additionally, Lyapunov exponents are tightly related to the geometry and topology of the energy surfaces [16].

\subsection{Integration algorithm}

It is well known that in order to faithfully represent the true trajectories of a Hamiltonian flow by means of numerical pseudo-trajectories one has to resort to symplectic algorithms. The simplest one is the leap-frog algorithm which, for the Hamiltonian of interest of the form

$$
H(\boldsymbol{\theta}, \mathbf{p})=\sum_{i=1}^{N} \frac{p_{i}^{2}}{2}+V(\boldsymbol{\theta})
$$

reads as

$$
\begin{aligned}
& \theta_{i}(t+\Delta t)=\theta_{i}(t)+\Delta t p_{i}(t) \\
& p_{i}(t+\Delta t)=p_{i}(t)-\Delta t \bar{\nabla}_{i} V[\boldsymbol{\theta}(t+\Delta t)],
\end{aligned}
$$

where $\bar{\nabla} V=\left(\frac{\partial}{\partial \theta_{1}} \ldots \frac{\partial}{\partial \theta_{N}}\right)$. In order to work with a more precise integration scheme, we used a second order bilateral symplectic algorithm [27], i.e.

$$
\begin{aligned}
& \left(\boldsymbol{\theta}^{(0)}, \mathbf{p}^{(0)}\right)=[\boldsymbol{\theta}(t), \mathbf{p}(t)] \\
& \theta_{i}^{(1)}=\theta_{i}(t) \\
& p_{i}^{(1)}=p_{i}(t)-\frac{1}{2} \Delta t \bar{\nabla}_{i} V\left[\boldsymbol{\theta}^{(1)}\right] \\
& \theta_{i}^{(2)}=\theta_{i}^{(1)}+\Delta t p_{i}^{(1)} \\
& p_{i}^{(2)}=p_{i}^{(2)}-\frac{1}{2} \Delta t \bar{\nabla}_{i} V\left[\boldsymbol{\theta}^{(2)}\right] \\
& p_{i}^{(3)}=p_{i}^{(2)} \\
& \theta_{i}^{(3)}=\theta_{i}^{(2)}+\frac{1}{2} \Delta t p_{i}^{(3)} \\
& p_{i}^{(4)}=p_{i}^{(3)}-\Delta t \bar{\nabla}_{i} V\left[\boldsymbol{\theta}^{(3)}\right] \\
& \theta_{i}^{(4)}=\theta_{i}^{(4)}+\Delta t p_{i}^{(3)} \\
& {[\boldsymbol{\theta}(t+2 \Delta t), \mathbf{p}(t+2 \Delta t)]=\left(\boldsymbol{\theta}^{(4)}, \mathbf{p}^{(4)}\right) .}
\end{aligned}
$$

Symplectic algorithms compute the time evolution of the coordinates, that is $\left\{p_{i}(t), q_{i}(t)\right\} \rightarrow\left\{p_{i}(t+\Delta t), q_{i}(t+\Delta t)\right\}$, by performing a canonical transformation at each time step [28], therefore all the Poincaré invariants in phase space are conserved, in particular energy conservation and phase space volumes conservation (Liouville 
theorem) are fulfilled. Energy fluctuations $\Delta E / E$ can be made arbitrarily small by reducing the integration time step. Any other kind of integration scheme, no matter if of high order, would not faithfully represent a Hamiltonian flow. We used a bilateral scheme (which takes into account the exchangeability of the $\left\{p_{i}(t)\right\}$ and $\left.\left\{q_{i}(t)\right\}\right)$ under a suitable canonical transformation) because for any given $\Delta t$ it is more precise with respect to the basic scheme in equation (16).

\subsection{Initialization of the system}

The system was initialized by setting the total energy equal to some given value $E$ and by setting the total angular momentum $P$, without loss of generality, equal to zero, i.e. $P=\mathscr{P}=0$.

To do so, we first considered the initial value of each spin $\theta_{i j}$ as a random variable uniformly distributed in the interval $[0,2 \pi \alpha]$, where $0<\alpha<1$. Then, we computed the corresponding potential energy value $V(\theta)$. If this was found larger than the value of the initially assigned total energy $E$, then the variables $\left\{\theta_{i j}\right\}$ were tentatively rescaled through a new parameter $\alpha_{\text {new }}<\alpha$. This procedure was iterated until the condition $E-V=K>0$ was fulfilled (where $K$ is the kinetic energy). Then the momenta were initialized by dividing them into two sets, say $A$ and $B$, and choosing them randomly to satisfy the condition $\sum_{i j \in A} p_{i j}^{2}=K$. This is obtained by choosing a unit vector $v$ on

the $\mathscr{S}^{(N / 2-1)}$ sphere, and then setting $p_{i j}=\sqrt{2 K} v_{i j}$ (where $v_{i j}$ are the components of the unit vector $v$ ). Finally, to impose the condition $\mathscr{P}=0$, we chose the momenta in the set $B$ equal and opposite to those of the set $A: p_{i, j+N / 2}=-p_{i, j}$, where $i \in[1, N]$ and $j \in[1, N / 2]$. The dynamics of the $X Y 2 \mathrm{D}$ model is chaotic, as it has been reported in reference [24], and thus, being a non-integrable system, after the Poincaré-Fermi theorem the system has no non-trivial constant of motion besides energy. Hence the dynamics is bona fide ergodic and mixing so that there is no dependence of time averages on the initial conditions, provided that the integration time is sufficiently long to observe a good convergence of the time averages. Nevertheless, in what follows, to reduce as much as possible the errors on the numerical outcomes we have performed a double averaging, that is, in time and on different initial conditions randomly sampled on any given hypersurface of constant energy and vanishing total momentum.

\subsection{Numerical evidence of the $\mathrm{KT}$ phase transition}

Numerical integration of the Hamiltonian dynamics of the system was performed by setting $J=1$ and time step $\Delta t=10^{-3}$. After a transient of $10^{5}$ time steps to make the system thermalise, the averages of any observable have been computed by considering a total number of steps equal to $2 \times 10^{6}$, and 250 different trajectories. The results obtained have very small fluctuations so that in the figures that follow error bars are not shown because are always smaller than the size of the symbols.

Microcanonical averages were computed through arithmetic averages of the sequences of values taken by any given observable along the dynamical trajectories, 
Geometrical and topological study of the Kosterlitz-Thouless phase transition in the $\mathbf{X Y}$ model in two dimensions
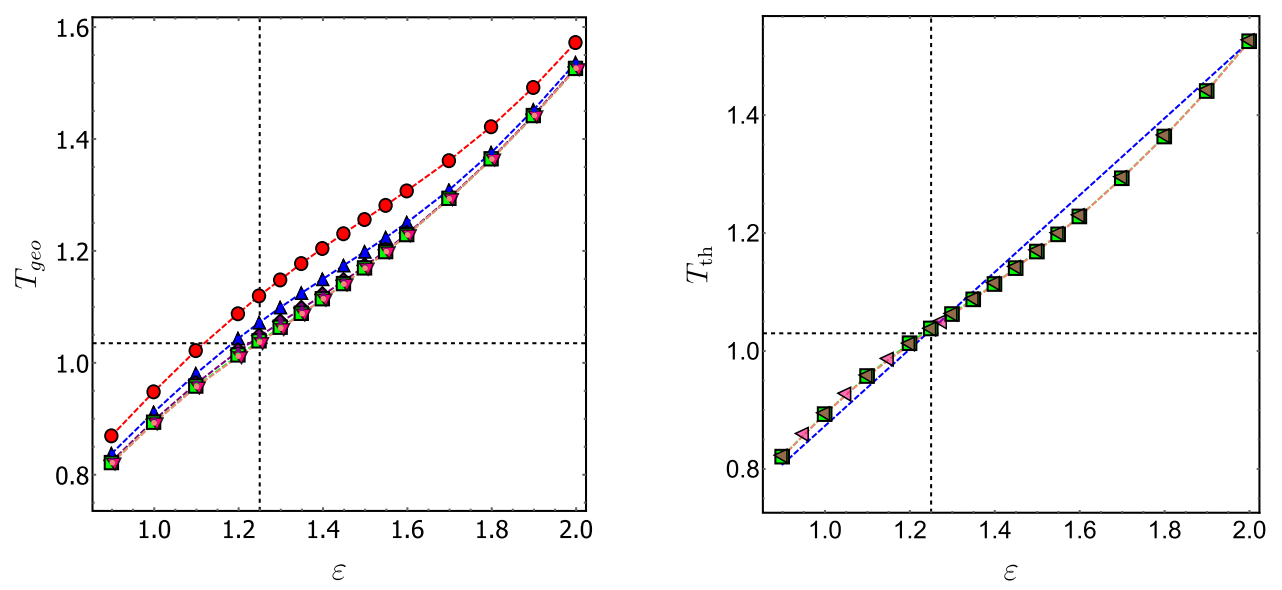

Figure 2. Caloric curves. (Left) Geometrical temperature vs specific energy from equation (8), $N=6 \times 6$ (red circles), $N=10 \times 10$ (blue triangles), $N=20 \times 20$ (purple diamonds), $N=40 \times 40$ (green squares), $N=70 \times 70$ (pink triangles). (Right) The thermodynamic temperature $T_{\text {th }}$ from equation (19) versus energy density $\varepsilon$ is reported only for $N=40 \times 40$ (green squares) and $N=70 \times 70$ (pink triangles) to put in evidence the inflection point through the blue dashed line. For both panels the vertical dotted line marks the transition point at $\varepsilon_{c}=1.25$. The horizontal dotted line identifies the critical temperature $T_{c}=1.03$.

that is, if $A_{j}(i)$ is the value of the observable $A$ at the $i$ th step along the $j$ th trajectory

$$
\langle A\rangle_{\mu c}=\frac{1}{N_{\mathrm{trj}}} \sum_{j=1}^{N_{\mathrm{trj}}}\left(\frac{\sum_{i=1}^{N_{\mathrm{step}}} A_{j}(i)}{N_{\mathrm{steps}}}\right),
$$

where $N_{\text {trj }}$ is the number of trajectories and $N_{\text {step }}$ is the number of integration steps. In order to locate the phase transition, we have computed the caloric curve (thermodynamic temperature $T_{\text {th }}$ versus energy density $\varepsilon=E / N$ ) and the constant volume specific heat.

The microcanonical expressions for these quantities are developed in [29], based on a method proposed in [30], and obtained in the form

$$
T_{\mathrm{th}}=\left[\left(\frac{1}{2}-\frac{3}{2 N}\right)\left\langle\kappa^{-1}\right\rangle_{\mu c}\right]^{-1} .
$$

for the thermodynamic temperature, and

$$
c_{v}=\frac{1}{N}\left[1-\frac{\left(1-\frac{5}{N}\right)\left\langle\kappa^{-2}\right\rangle_{\mu c}}{\left(1-\frac{3}{N}\right)\left\langle\kappa^{-1}\right\rangle_{\mu c}^{2}}\right]^{-1} .
$$

for the specific heat, where $\kappa=K / N$ is the specific kinetic energy.

In figures 2 and 3 , the results for the specific heat and the geometrical and thermodynamic temperature as functions of the specific energy have been reported. 


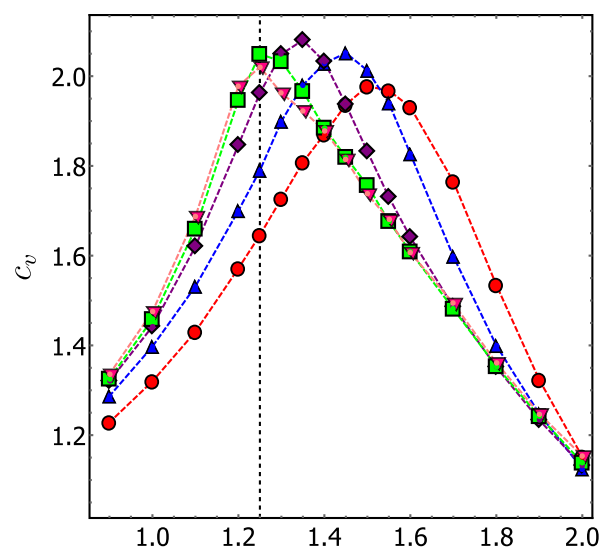

Figure 3. Specific heat vs energy density $\varepsilon$, for different lattice dimensions: $N=$ $6 \times 6$ (red circles), $N=10 \times 10$ (blue triangles), $N=20 \times 20$ (purple diamonds), $N=40 \times 40$ (green squares), $N=70 \times 70$ (pink triangles). The vertical dotted line marks the transition point $\varepsilon_{c}$.

As a matter of fact, we are tackling the KT phase transition in the framework of the microcanonical ensemble because the microcanonical measure is the ergodic invariant measure for a non-integrable Hamiltonian flow. Therefore, we identify the phase transition point by following a recent and brilliant classification scheme of $\mathrm{PT}$ in the microcanonical ensemble given by Qi and Bachmann [31, 32]. In the absence of an order parameter - as is the case of the 2D $X Y$ model - this consists of locating the inflection point of the caloric curve. In so doing, the inflection point of $T_{\text {th }}(\varepsilon)$ reported in figure 2 identifies the phase transition point at the energy density $\varepsilon_{c}=1.25$ in perfect agreement with the peak of the specific heat reported in figure 3 (where the points obtained with lattices of $40 \times 40$ and $70 \times 70$ sites superpose). This agreement is consistent with the fact that the caloric curve $T=T(E)$ and the specific heat are related by $C_{v}=(\partial T / \partial E)^{-1}$. Through the caloric curve we obtain $T_{c}=T\left(\varepsilon_{c}=1.25\right) \simeq 1.03$.

Other phenomenological indicators of the transition that we have computed, and reported in figure 4, are the derivatives of the specific volume $\rho_{N}=$ $\left[\Omega_{N}(N \varepsilon) / \Omega_{N}\left(N \varepsilon_{\min }\right)\right]^{1 / N}$ where $\Omega_{N}(N \varepsilon)$ is the volume (see equation (54))

$$
\Omega_{N}(N \varepsilon)=\int_{\Sigma_{E, \mathscr{P}}} \frac{\mathrm{d} \sigma_{\Sigma_{E, \mathscr{P}}}}{\sqrt{\mathbb{G}(\nabla H, \nabla P)}}
$$

and $\Omega_{N}\left(N \varepsilon_{\min }\right)$ is the microcanonical volume at the lowest value (arbitrary) of the energy density considered so that $\rho_{N}\left(\varepsilon_{\min }\right)=1$. Thus, apart from a constant depending on the choice of $\varepsilon_{\min }$, the microcanonical specific entropy is $S_{N}(\varepsilon)=\log \rho_{N}(\varepsilon)$, so that $\partial \rho_{N} / \partial \varepsilon=$ $\rho_{N}(\varepsilon)\left(\partial S_{N} / \partial \varepsilon\right)=\rho_{N}(\varepsilon) \beta(\varepsilon)$, where $\beta(\varepsilon)$ is the inverse of temperature of equation (19). This quantity measures the way the density of energy-states varies as a function of the energy. Also this function displays an inflection point at $\varepsilon_{c}=1.25$. 
Geometrical and topological study of the Kosterlitz-Thouless phase transition in the $\mathbf{X Y}$ model in two dimensions
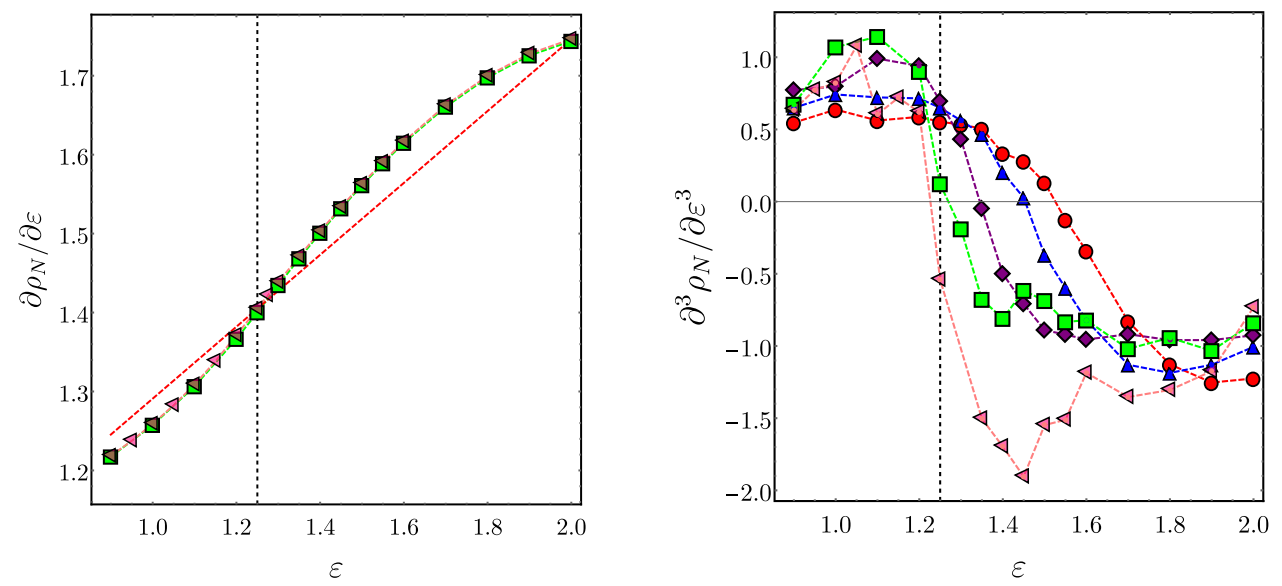

Figure 4. (Left) The first derivative of the density of states $\partial \rho_{N} / \partial \varepsilon$ versus energy density $\varepsilon$ displays an inflection point at the transition energy density $\varepsilon_{c}=1.25$. (Right) The third derivative of the density of states tends to develop a step-like pattern at increasing $N . N=6 \times 6$ (red circles), $N=10 \times 10$ (blue triangles), $N=20 \times 20$ (purple diamonds), $N=40 \times 40$ (green squares), $N=70 \times 70$ (pink triangles).

Another interesting quantity is the third derivative of the specific volume

$$
\frac{\partial^{3} \rho_{N}(\varepsilon)}{\partial \varepsilon^{3}}=\rho_{N}(\varepsilon)\left[\frac{\partial^{3} S_{N}}{\partial \varepsilon^{3}}+3 \frac{\partial S_{N}}{\partial \varepsilon} \frac{\partial^{2} S_{N}}{\partial \varepsilon^{2}}+\left(\frac{\partial S_{N}}{\partial \varepsilon}\right)^{3}\right]
$$

which is computed through the time averages of $\left\langle\kappa_{N}^{-n}\right\rangle[29]$ entering the derivatives of the entropy

$$
\begin{aligned}
\frac{\partial S_{N}}{\partial \varepsilon} & =\left(\frac{1}{2}-\frac{3}{2 N}\right)\left\langle\kappa_{N}^{-1}\right\rangle_{\mu c} \\
\frac{\partial^{2} S_{N}}{\partial \varepsilon^{2}} & =N\left[\left(\frac{1}{2}-\frac{3}{2 N}\right)\left(\frac{1}{2}-\frac{5}{2 N}\right)\left\langle\kappa_{N}^{-2}\right\rangle_{\mu c}-\left(\frac{1}{2}-\frac{3}{2 N}\right)^{2}\left\langle\kappa_{N}^{-1}\right\rangle_{\mu c}^{2}\right] \\
\frac{\partial^{3} S_{N}}{\partial \varepsilon^{3}} & =N^{2}\left(\frac{1}{2}-\frac{3}{2 N}\right)\left(\frac{1}{2}-\frac{5}{2 N}\right)\left(\frac{1}{2}-\frac{7}{2 N}\right)\left\langle\kappa_{N}^{-3}\right\rangle_{\mu c}+ \\
& \left.-3\left(\frac{1}{2}-\frac{3}{2 N}\right)^{2}\left(\frac{1}{2}-\frac{5}{2 N}\right)\left\langle\kappa_{N}^{-1}\right\rangle_{\mu c}\left\langle\kappa_{N}^{-2}\right\rangle_{\mu c}+2\left(\frac{1}{2}-\frac{3}{2 N}\right)^{3}\left\langle\kappa_{N}^{-1}\right\rangle_{\mu c}^{3}\right],
\end{aligned}
$$

and by deriving $\rho_{N}$ from the numerical integration of the function $\partial_{\epsilon} S_{N}$, i.e.

$$
\rho_{N}(\epsilon)=\rho_{N}\left(\epsilon_{\min }\right) \exp \left[\int_{\epsilon_{\min }}^{\epsilon} \frac{\partial S_{N}}{\partial \varepsilon}(\epsilon)\right] \mathrm{d} \varepsilon=\rho_{N}\left(\epsilon_{\min }\right) \exp \left[\int_{\epsilon_{\min }}^{\epsilon}\left(\frac{1}{2}-\frac{3}{2 N}\right)\left\langle\kappa_{N}^{-1}\right\rangle_{\text {time }}\right] \mathrm{d} \varepsilon .
$$

As is shown in figure $4, \partial^{3} \rho_{N} / \partial \varepsilon^{3}$ displays a remarkable property, that is, at increasing $N$ its $\varepsilon$-shape tends to develop a step-like pattern. This is suggestive of the divergence 
Geometrical and topological study of the Kosterlitz-Thouless phase transition in the $\mathbf{X Y}$ model in two dimensions

of the fourth derivative of $\rho_{N}(\varepsilon)$ as $N \rightarrow \infty$. In other words, and within the limits of numerical simulations, there is also a quantity which is singular at $\varepsilon_{c}=1.25$.

On the other hand, as already stated above, the common way of numerically tackling the phase transition in the 2D $X Y$ model is in the framework of canonical ensemble. By means of Monte Carlo canonical simulations the critical temperature of the transition is fitted through the divergence of the spatial correlation length $\xi \sim$ $\exp \left\{b\left[\left(T-T_{\mathrm{c}}\right) / T_{\mathrm{c}}\right]^{-1 / 2}\right\}$ in the spin-spin correlation function $\left\langle\mathbf{s}_{i} \cdot \mathbf{s}_{j}\right\rangle \sim \exp (-a|i-j| / \xi)$ where $a$ and $b$ are constants. Even though there has been some controversy about the extent of the critical region, as was discussed in a thorough analysis in reference [33] confirmed in reference [34], the transition temperature is fixed at $k_{\mathrm{B}} T_{\mathrm{c}} / J=0.8929$, in excellent agreement with early findings of $k_{\mathrm{B}} T_{\mathrm{c}} / J \simeq 0.894$ reported in [35-37]. While we estimate a higher transition temperature with respect to this value, it is worth mentioning that results worked out by means of nonperturbative renormalization group in [38] gave $0.9<k_{\mathrm{B}} T_{\mathrm{c}} / J<1$ for the critical temperature, and by means of functional renormalization in [39] the authors found $0.91<k_{\mathrm{B}} T_{\mathrm{c}} / J<1.02$. Both of these intervals of values are reasonably compatible with our estimate of $T_{\mathrm{c}} \simeq 1.03$. Last but not least, the peak of specific heat that we report at $T \simeq 1.03$ is in agreement with old and recent findings in references [35-37, 39].

As a final remark on the estimate of the transition temperature, finding the energy at which the pairs of vortices unbind in order to locate the transition as is commonly done in Monte Carlo simulations, would have - as a consequence - that the correspondence with what we are after in the present work (the topological origin of the KT transition) would be lost. Equations (27) and (28) relate microcanonical entropy $S(E)$ with topological invariants of the submanifolds of phase space $M_{E}$ and $\Sigma_{E}$ respectively, therefore we needed to locate the phase transition through entropy-related quantities: $T=T(E)$, since $T(E)=(\partial S / \partial E)^{-1}$, and specific heat, since $C_{v}=-(\partial S / \partial E)^{2}\left(\partial^{2} S / \partial E^{2}\right)^{-1}$, and $\partial^{3} \rho_{N}(\varepsilon) / \partial \varepsilon^{3}$. In this way the results reported in the following are consistent with our estimate of $\varepsilon_{c}=E_{c} / N=1.25$.

\section{Numerical results on the geometry and the topology of the submanifolds $\Sigma_{\mathrm{E}, 0}$ in the $X Y-2 D$ model}

In the case of geometrical observables we are interested in the computation of the averages with respect to the Riemannian induced measure $\sigma_{\Sigma_{E, 0}}$, i.e.

$$
\langle A\rangle_{\text {geo }}=\frac{\int_{\Sigma_{E, 0}} A \sigma_{\Sigma_{E, 0}}}{\int_{\Sigma_{E, 0}} \sigma_{\Sigma_{E, 0}}} .
$$

As the numerical simulation of the Hamiltonian dynamics allows to sample the submanifold $\Sigma_{E, 0}$ according to the microcanonical measure, we have to express the geometric averages (24) as functions of microcanonical averages computed according to the equation (18). As shown in [40], the geometrical averages can be estimated as follows (see equation (55) of appendix A.2)

$$
\langle A\rangle_{\mathrm{geo}}=\frac{\left\langle A \chi^{-1}\right\rangle_{\mu c}}{\left\langle\chi^{-1}\right\rangle_{\mu c}}
$$




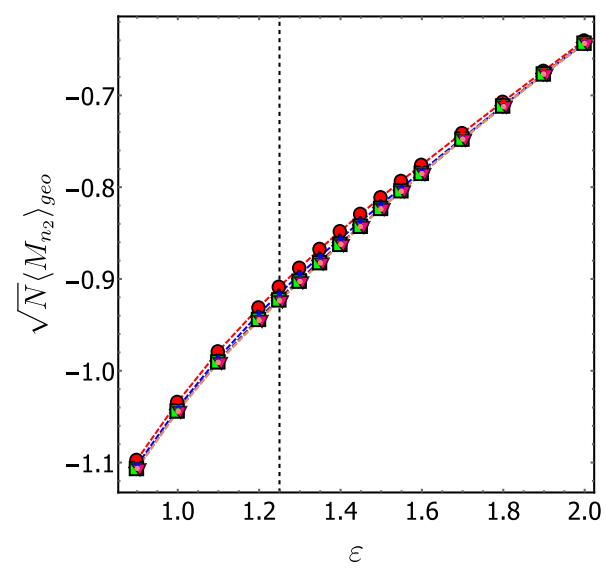

Figure 5. Geometrical average of the mean curvature for for $N=6 \times 6$ (red circles), $N=10 \times 10$ (blue triangles), $N=20 \times 20$ (purple diamonds), $N=40 \times 40$ (green squares), $N=70 \times 70$ (pink triangles). The vertical dotted line marks the transition point $\varepsilon_{c}$.

First we have computed - by means of the geometrical measure - what has been defined a basic quantity characterizing the extrinsic geometry of the energy level sets, that is, the total mean curvature $\sqrt{N}\left\langle M_{n_{2}}\right\rangle$ as a function of the specific energy. Since the average of the mean curvature (i.e. the total mean curvature) is not an intensive observable, the factor $\sqrt{N}$ allows to compare the curves obtained at different $N$. The results are displayed in figure 5 . The $\varepsilon$-pattern of $\sqrt{N}\left\langle M_{n_{2}}\right\rangle$ displays a concavity change at the transition point. At $\varepsilon>\varepsilon_{c}$ the values of $\sqrt{N}\left\langle M_{n_{2}}\right\rangle$ fall on a straight line, whereas at $\varepsilon<\varepsilon_{c}$ they fall on a curve. However, this provides a weak geometric signal at the transition point, reflecting the somewhat weak thermodynamic signal shown by the caloric curve. Sharper signals of the transition can be obtained by resorting to observables that are to some extent sensitive to the details of the geometric fabric of the energy level sets. Actually, this is what is observed by means of the variance of the mean curvature reported in figure 6 .

Here the change of concavity of $\sigma_{\text {geo }}^{2}\left(M_{n_{2}}\right)$, and thus of an abrupt variation of its second derivative with respect to $\varepsilon$, is definitely more marked than in the case of the average mean curvature. A sharpening with $N$ of this effect is also very clear, what appears as an inflection point for the smaller lattice sizes considered, then seems to evolve towards a 'corner' point at larger $N$. The variance of the mean curvature is a measure of the deviation from isotropy of a manifold, loosely speaking a measure of the degree of 'bumpiness' of its landscape and of the presence of high dimensional 'holes'.

However, the geometric observable which is of central interest in the present work is the average variance of the principal curvatures of the $\Sigma_{E, 0}$, that is, $\left\langle\sigma^{2}\left(k_{i}\right)\right\rangle_{\text {geo }}$, reported in figures 7 and 8. As explained in section 3, after Pinkall's theorem this observable makes the link between geometry and topology of the $\left\{\Sigma_{E, 0}\right\}_{E \in \mathbb{R}}$ foliating phase space. A peculiar $\varepsilon$-change of this geometric property of the manifolds $\left\{\Sigma_{E, 0}\right\}_{E \in \mathbb{R}}$, occurring at the $\varepsilon_{c}$ which pinpoints the KT phase transition, is the 'shadow' of a deeper phenomenon: a peculiar $\varepsilon$-change of the topology of these manifolds. An upstream phenomenon at the 
Geometrical and topological study of the Kosterlitz-Thouless phase transition in the $\mathbf{X Y}$ model in two dimensions

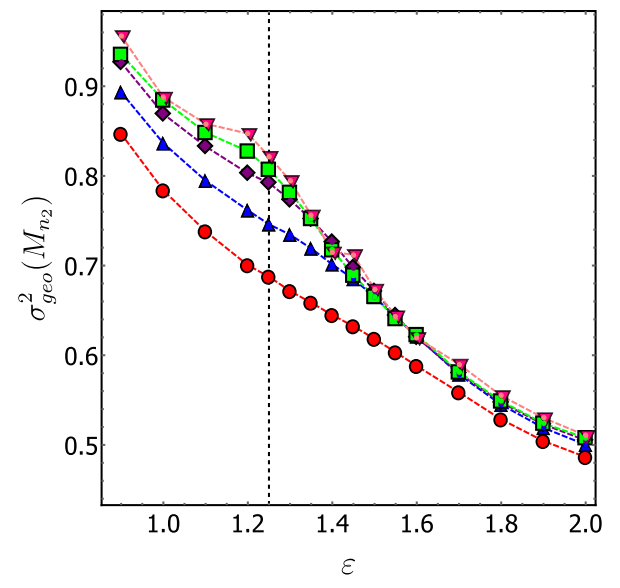

Figure 6. Geometrical variance of the mean curvature for $N=6 \times 6$ (red circles), $N=10 \times 10$ (blue triangles), $N=20 \times 20$ (purple diamonds), $N=40 \times 40$ (green squares), $N=70 \times 70$ (pink triangles). The vertical dotted line marks the transition point $\varepsilon_{c}$.

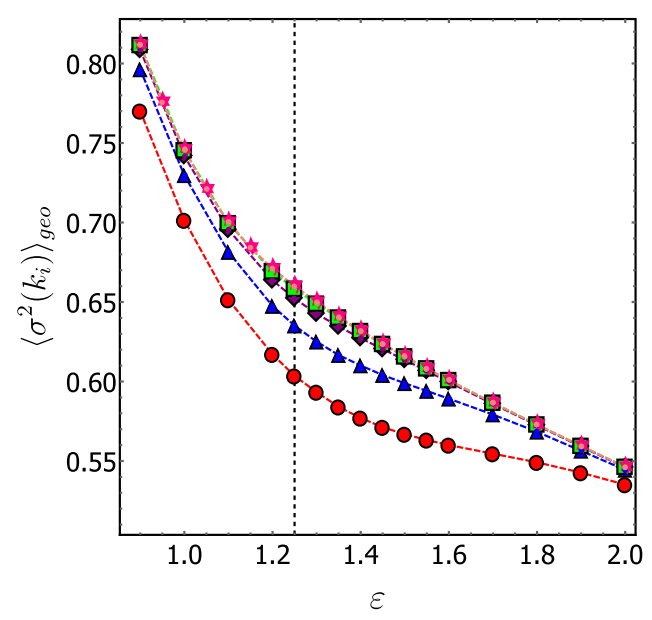

Figure 7. Lattice size dependence of the geometrical average of the dispersion of the principal curvatures for $N=6 \times 6$ (red circles), $N=10 \times 10$ (blue triangles), $N=20 \times 20$ (purple diamonds), $N=40 \times 40$ (green squares), $N=70 \times 70$ (pink triangles). The vertical dotted line marks the transition point $\varepsilon_{c}$.

very grounds of a phase transition, at variance with the common viewpoint attributing the origin of PT to a loss of analyticity of statistical measures.

Figure 8-where only the outcomes worked out for the two largest sizes of the lattice are reported-clearly shows that for $\varepsilon>\varepsilon_{c}$ the values of $\left\langle\sigma^{2}\left(k_{i}\right)\right\rangle_{\text {geo }}$ are reasonably well fitted by a segment of a straight line, thus $\partial_{\varepsilon}^{2}\left\langle\sigma^{2}\left(k_{i}\right)\right\rangle_{\text {geo }}=0$ within the limits of the numerical computation. To the contrary, for $\varepsilon<\varepsilon_{c}$ it is well evident that $\partial_{\varepsilon}^{2}\left\langle\sigma^{2}\left(k_{i}\right)\right\rangle_{\text {geo }}>0$. The overall $\varepsilon$-pattern of $\partial_{\varepsilon}^{2}\left\langle\sigma^{2}\left(k_{i}\right)\right\rangle_{\text {geo }}$ is illustrated by the lower panel of figure 8 where this function is obtained by numerically deriving twice the points reported 
Geometrical and topological study of the Kosterlitz-Thouless phase transition in the $\mathbf{X Y}$ model in two dimensions
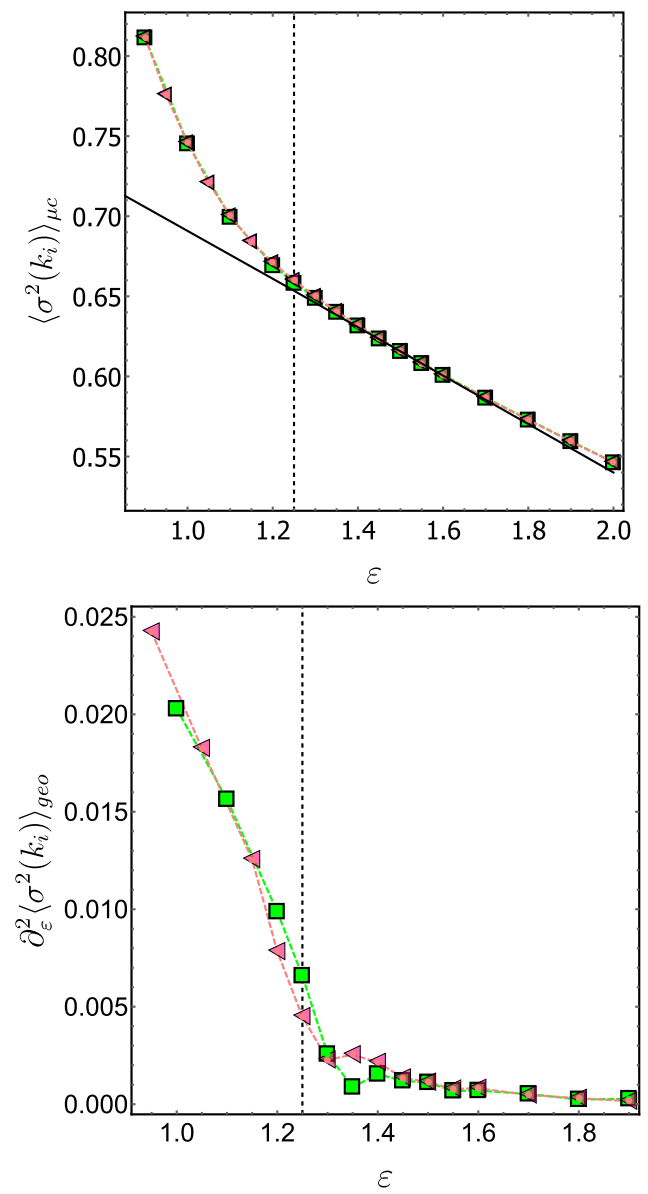

Figure 8. (Top) Geometrical average of the dispersion of the principal curvatures for $N=40 \times 40$ (green squares), and $N=70 \times 70$ (pink triangles). (Bottom) Numerical second order derivative of the data of the upper panel. The vertical dotted line marks the transition point $\varepsilon_{c}$.

in the upper panel of the same figure according to the expression $\partial_{\varepsilon}^{2}\left\langle\sigma^{2}\left(k_{i}\right)\right\rangle\left(\varepsilon_{i}\right)=$ $\left[\left\langle\sigma^{2}\left(k_{i}\right)\right\rangle\left(\varepsilon_{i}+\Delta \varepsilon\right)+2\left\langle\sigma^{2}\left(k_{i}\right)\right\rangle\left(\varepsilon_{i}\right)-\left\langle\sigma^{2}\left(k_{i}\right)\right\rangle\left(\varepsilon_{i}-\Delta \varepsilon\right)\right] /(\Delta \varepsilon)^{2}$. Again within the limits of numerical computations, the results are suggestive of the presence of an angular point close to the transition energy density. The larger the lattice size the closer the 'singular' point of $\partial_{\varepsilon}^{2}\left\langle\sigma^{2}\left(k_{i}\right)\right\rangle_{\text {geo }}$ to $\varepsilon_{c}$.

Figure 8 , and notably its lower panel, shows a marked geometrical signal of topological meaning of the KT transition. This is already what we aimed at in the present work.

This notwithstanding, further consequences can be drawn from the main result reported in figure 8 .

According to Pinkall's theorem, a bifurcation of the second derivative of the topological term [41] in the right-hand side of equation (13) can entail the bifurcation of the second derivative of the left-hand side of the same equation. 
Then, in order to make a link between the topological signature and a thermodynamical signature of the KT phase transition, let us resort to the existence of a relationship between thermodynamic entropy and phase space topology provided by the following exact formula $[16]$

$$
\begin{aligned}
S_{N}^{(-)}(E) & =\left(k_{\mathrm{B}} / N\right) \log \left[\int_{M_{E}^{N}} \mathrm{~d}^{N} p \mathrm{~d}^{N} q\right] \\
& =\frac{k_{\mathrm{B}}}{N} \log \left[\operatorname{vol}\left[M_{E}^{N} \backslash \bigcup_{i=1}^{N(E)} \Gamma\left(x_{c}^{(i)}\right)\right]+\sum_{i=0}^{N} w_{i} \mu_{i}\left(M_{E}^{N}\right)+\mathscr{R}\right]
\end{aligned}
$$

where $\mu_{i}\left(M_{E}^{N}\right)$ are the Morse indexes (in one-to-one correspondence with topology changes) of the submanifolds $\left\{M_{E}^{N}=H^{-1}((-\infty, E])\right\}_{E \in \mathbb{R}}$ of phase space; in square brackets: the first term is the result of the excision of certain neighbourhoods of the critical points of the Hamiltonian from $M_{E}^{N}$; the second term is a weighed sum of the Morse indexes, and the third term is a smooth function of $N$ and $E$. Another way of relating thermodynamical entropy with topology, in this case of $\Sigma_{E}^{N}$ in place of $M_{E}^{N}$, leads to the approximate formula

$$
S_{N}(E)=\frac{k_{\mathrm{B}}}{N} \log \int_{\Sigma_{E}^{N}} \frac{\mathrm{d} \mu}{\|\nabla H\|} \simeq \frac{k_{\mathrm{B}}}{N} \log \left[\operatorname{vol}\left(\mathbb{S}_{1}^{N-1}\right) \sum_{i=0}^{N} b_{i}\left(\Sigma_{E}^{N}\right)+\mathscr{R}_{1}(E)\right]+\mathscr{R}_{2}(E),
$$

where $\mathscr{R}_{1}(E)$, and $\mathscr{R}_{2}(E)$ are smooth functions, and $b_{i}\left(\Sigma_{E}^{N}\right)$ are the Betti numbers of the energy level sets, and $\mathrm{d} \mu$ is the measure on the level set.

We see that the variation with $E$ of the topology of the $\Sigma_{E}^{N}$ implies also the variation with $E$ of the total entropy, independently of its definition, that is, equation (26) versus equation (28). Hence we see that, in general, some adequate variation of the topology of the $\Sigma_{E}^{N}$ foliating the phase space of a given system is sufficient to induce a phase transition. In principle, different kinds of topology variations can induce PT of different kinds. More precisely, after the microcanonical definition of the constant volume specific heat $1 / C_{v}=\partial T(E) / \partial E$, where $T^{-1}=\partial S(E) / \partial E$, we have

$$
C_{v}=-\left(\frac{\partial S}{\partial E}\right)^{2}\left(\frac{\partial^{2} S}{\partial E^{2}}\right)^{-1}
$$

so that, in the case of the KT phase transition, the cuspy pattern of the specific heat reported in figure 3 is necessarily related to the energy dependence of the second derivative of the entropy, since the first derivative appears rather regular after figure 2 reporting $(\partial S(E) / \partial E)^{-1}$.

Thus, if the second derivative with respect to the energy of any combination of the Betti numbers of the $\Sigma_{E}^{N}$, or of the Morse indexes of the $\mu_{i}\left(M_{E}^{N}\right)$, makes a sharp change at some value of the energy [41], then this affects both the second derivatives with respect to the energy of $\left\langle\sigma^{2}\left(k_{i}\right)\right\rangle_{\text {geo }}(\varepsilon)$ and of the entropy.

Actually, as is shown in figure 9, the $\varepsilon$ dependence of the second derivative of the entropy is strongly reminiscent of the $\varepsilon$ dependence of the second derivative of $\left\langle\sigma^{2}\left(k_{i}\right)\right\rangle_{\text {geo }}$, 


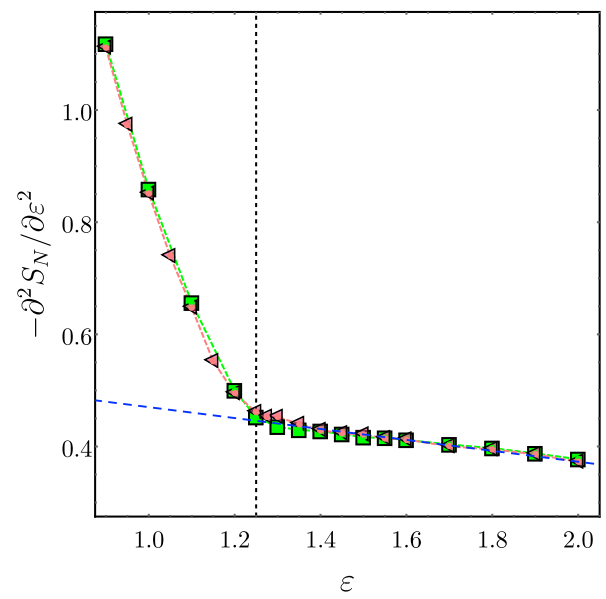

Figure 9. Second order derivative of the entropy versus energy density. Lattice sizes: $N=40 \times 40$ (green squares), and $N=70 \times 70$ (pink triangles).

a similarity which is not accidental in the light of the above given arguments. In fact, even if a rigorous detailed relation has not yet been worked out, a sudden change in the way of changing of topology with energy is a sufficient condition to entail the same kind of variation of the entropy, that is, of its second $\varepsilon$ derivative. In conclusion, the deep origin of the KT phase transition is of topological kind also when tackled from the viewpoint of phase space topology.

\section{Discussion}

The KT phase transition has been studied in the framework of the microcanonical ensemble. As is well known, this kind of transition does not correspond to a global symmetry breaking so that the standard Landau classification does not apply. A possible way of going beyond the phenomenological level of Landau theory can be achieved by focussing on the change of geometrical and topological properties of the constant energy hypersurfaces which foliate the phase space of an $X Y-2 \mathrm{D}$ model. This approach has been already proved effective when applied to a simple gauge model also lacking a global symmetry breaking and thus an order parameter [18].

According to an appropriate classification of PT in the microcanonical ensemble [31], the inflection point in the caloric curve and the peak of the specific heat have been used to locate the phase transition by numerically solving the Hamilton equations of motion. Then the variations of the extrinsic geometry of the energy level sets have been numerically investigated across $\varepsilon_{c}$, the critical specific energy of the phase transition point. The conceptual consequence of this analysis is that the thermodynamic transitional phenomenology has its origin at the more fundamental level of the geometrical changes of the energy level sets of phase space. In turn, these geometry changes are probes of deeper changes of the topology of the same energy level sets, as per a theorem in differential topology.

The KT phase transition has been a paradigmatic topic of a successful application of topological concepts to broaden the theoretical understanding of PT. In our present work 
Geometrical and topological study of the Kosterlitz-Thouless phase transition in the $\mathbf{X Y}$ model in two dimensions

we have again tackled this topic with the aid of topological tools. However, at variance with the original approach focussing on topological defects in real physical space, here we have focussed on the topology of subsets of phase space. The highly non trivial bridge between these two levels of description can be traced to the correspondence between topological defects in real space (spin vortices) and critical points of the potential part of the Hamiltonian.

An attempt in this direction has already been reported in reference [19], however, as discussed by the authors, the results were not conclusive because of the hard numerical task of finding all the critical points of the potential of the 2D $X Y$ model, or of locating at least a relevant subset of them. Another interesting and original approach to the study of topological aspects of PT in classical spin models has been put forward in [42]. Though applied to two exactly solvable versions of the $X Y$ model, that is its one-dimensional version and its so-called mean-field version, in this paper the authors propose an intriguing approach resorting to the so-called integral calculus based on the Euler characteristic and on the definition of an 'Euler entropy' from which they derive an 'Euler temperature' which turns out to be identical to the thermodynamic temperature at the phase transition point, thus providing a topology-based method to compute the critical temperature of a phase transition.

Finally, in a very recent paper [43], persistent homology, a constructive method in algebraic topology, has been used to tackle PT in spin systems. The authors confirm a topological origin of the $\mathrm{KT}$ transition of the 2D $X Y$ model, though they considered topological aspects related with real space vortex-antivortex pairs rather than topology of configurations space. This work provides an independent confirmation of the relevance of topology for the KT transition and also confirms that the use of persistent homology is worthwhile to constructively investigate the topological origin of PT in a broad variety of systems as was first shown in [44].

\section{Acknowledgments}

This work has been done within the framework of the Master thesis work of Ghofrane Bel Hadj Aissa performed under the supervision of Matteo Gori and Marco Pettini within the framework of the project MOLINT which has received funding from the Excellence Initiative of Aix-Marseille University-A*Midex, a French 'Investissements d'Avenir' Programme. Roberto Franzosi and Ghofrane Bel Hadj Aissa thank the support by the QuantERA, ERA-NET Co-fund 731473 (Project Q-CLOCKS), Italy. Matteo Gori thanks the financial support of DARPA (USA) for his long term visit at Howard University at Washington D.C.

\section{Appendix}

\section{A.1. Review of differential extrinsic geometry of codimension $m$ submanifolds}

The phase space can be regarded as a $2 N$ dimensional manifold where a set of coordinate $\left\{x^{\mu}\right\}_{\mu=1, \ldots, 2 N}$ can be chosen such that 
Geometrical and topological study of the Kosterlitz-Thouless phase transition in the $\mathbf{X Y}$ model in two dimensions

$$
\left\{\begin{array}{l}
x^{2 \nu-1}=p^{\tilde{\nu}} \quad \nu=1, \ldots, N \\
x^{2 \nu}=q^{\hat{\nu}} \quad \nu=1, \ldots, N
\end{array}\right.
$$

Given a set of smooth real functions $\left\{F^{i}\right\}_{i=1, \ldots, m}$ on the phase space $\Lambda$, with $m<2 N$, we define the level sets $\Sigma_{f^{1}, \ldots, f^{m}}$ as follows

$$
\Sigma_{f^{1}, \ldots, f^{m}}=\left\{x \in \Lambda \mid F^{k}(x)=f^{k} \quad \forall k=1, \ldots, m\right\} .
$$

If the following condition

$$
\mathrm{d} F^{1} \wedge \ldots \wedge \mathrm{d} F^{m} \neq 0
$$

holds for every point on $\Sigma_{f^{1}, \ldots, f^{m}}$ then this is a regular submanifold of codimension $m$. In order to give a Riemannian geometrical characterization of such a submanifold, a metric structure (metric tensor) $g$ has to be assigned over the phase space lambda $\Lambda$. In general this assignation can be quite arbitrary. Nevertheless for sake of consistency with the symplectic structure we should require that the Riemannian volume form

$$
\omega=\sqrt{\operatorname{det} g} \mathrm{~d} x^{1} \wedge \ldots \wedge \mathrm{d} x^{2 N}=\sqrt{\operatorname{det} g} \mathrm{~d} p^{1} \wedge \mathrm{d} q^{1} \ldots \wedge \mathrm{d} p^{N} \wedge \mathrm{d} q^{N}
$$

is the invariant volume form for the Hamiltonian system, i.e. $\mathscr{L}_{X_{\mathrm{H}}} \omega=0$. The simplest metric satisfying this condition is the Euclidean metric $g=\delta_{\mu \nu} \mathrm{d} x^{\mu} \otimes \mathrm{d} x^{\nu}$, i.e. the phase space is endowed with the structure of a Euclidean space $\mathbb{E}^{2 N}$. Thanks to the metric structure, it is possible to define the gradient $\nabla H$ of a function as a vector field such that

$$
g(\nabla H, X)=\imath_{X} \mathrm{~d} H
$$

that in components reads

$$
\imath_{\nabla H} \mathrm{~d} x^{\mu}=(\nabla H)^{\mu}=g^{\mu \nu} \frac{\partial H}{\partial x^{\nu}}=\partial^{\mu} H,
$$

A critical point of the function $H$ is a point such where the gradient is null $\nabla H=0$. Thanks to this definition, the condition (32) can be reformulated as the absence of critical points for any function $F^{k}$ and the linear independence of the $m$ gradient vector fields $\left\{\nabla F^{k}\right\}_{k=1, \ldots, m}$ for any points of $\Sigma_{f^{1}, \ldots, f^{m}}$. In each point of regular submanifold of codimesnsion $m$, the tangent bundle of the phase space splits into a tangent bundle to the submanifold $T \Sigma_{f^{1}, \ldots, f^{m}}$ and a normal bundle $N \Sigma_{f^{1}, \ldots, f^{m}}$

$$
\left.T \Lambda\right|_{\Sigma_{f^{1}, \ldots, f^{m}}}=T \Sigma_{f^{1}, \ldots, f^{m}} \oplus N \Sigma_{f^{1}, \ldots, f^{m}}
$$

where the normal bundle is defined by $N \Sigma_{f^{1}, \ldots, f^{m}}=\operatorname{Span}\left\{\nabla F^{1}, \ldots, \nabla F^{m}\right\}$. We define also an induced metric (or first fundamental form ) on such regular submanifold of codimension $m$, which is the assignment to each point of the inner product

$$
\langle,\rangle: \mathrm{T} \Sigma_{f^{1}, \ldots, f^{m}} \times \mathrm{T} \Sigma_{f^{1}, \ldots, f^{m}} \rightarrow \mathbb{R} .
$$

From the preceding discussion it follows that it is convenient to introduce an adapted orthonormal frame $\left\{e_{a}\right\}_{a=1, \ldots, 2 N}$ that allows to make explicit the split of the tangent 
Geometrical and topological study of the Kosterlitz-Thouless phase transition in the $\mathbf{X Y}$ model in two dimensions bundle, i.e. $e_{\bar{A}} \in \mathrm{N} \Sigma_{f^{1}, \ldots, f^{m}}$ with $\bar{A}=1, \ldots, m$ and $e_{A} \in \mathrm{T} \Sigma_{f^{1}, \ldots, f^{m}}$ with $A=m+$ $1, \ldots, 2 N$.

This is equivalent to define for each point a set of rotation matrix $\mathrm{e}_{a}^{\nu}$ that allows to pass from the coordinate natural frame $\left\{\partial_{1}, \ldots, \partial_{2 N}\right\}=\left\{\partial_{\nu}\right\}_{\nu=1, \ldots, 2 N}$ to the adapted coordinate orthonormal frame $\left\{e_{1}, e_{2}, \ldots, e_{2 N}\right\}=\left\{e_{a}\right\}_{a=1,2, \ldots, 2 N}$, i.e. $e_{a}=\mathrm{e}_{a}^{\nu} \partial_{\nu}$. In analogous way, the inverse matrix $e_{\nu}^{b}$ are defined allowing to pass from coordinates covectors $\left\{\mathrm{d} x^{1}, \ldots, \mathrm{d} x^{2 N}\right\}$ to adapted covector frame $\left\{\theta^{1}, \theta^{2}, \ldots, \theta^{2 N}\right\}$ (with $\imath_{e_{a}} \theta^{b}=\delta_{a}^{b}$ ), i.e. $\theta^{b}=e_{\mu}^{b} \mathrm{~d} x^{\mu}$.

In order to characterize the extrinsic geometry of the regular submanifold $\Sigma_{f^{1}, \ldots, f^{m}}$, for any normal vector field $n \in \mathrm{N} \Sigma_{f^{1}, \ldots, f^{m}}$, we introduce the shape operator $\mathscr{W}_{n}$ (called also the Weingarten operator) giving by the variation of $n$ along the directions tangent to the submanifold at any fixed point, i.e. $\mathscr{W}_{n}: T \Sigma_{f^{1}, \ldots, f^{m}} \rightarrow T \Sigma_{f^{1}, \ldots, f^{m}}$ s.t.

$$
\mathscr{W}_{n}(V)=-\left.\nabla_{V} n \quad V \in \mathrm{T} \Lambda\right|_{\Sigma_{f^{1}, \ldots, f m}},
$$

where $\nabla$ is the Levi Civita connection on $(\Lambda, g)$. Let us see first of all that $\operatorname{Im} \mathscr{W} \subseteq$ $T \Sigma_{f^{1}, \ldots, f^{m}}$, in fact

$$
g\left(\mathscr{W}_{n}(V), n\right)=-g\left(\nabla_{V} n, n\right)=-\frac{1}{2} \nabla_{V}[g(n, n)]=0
$$

so the Weingarten map can be rewritten in terms of the orthonormal vector basis

$$
\mathscr{W}_{n}\left(e_{B}\right)=\mathscr{W}_{n_{B}}^{A} e_{A}=-\nabla_{e_{B}} n \Rightarrow \mathscr{W}_{n_{B}}^{A}=-\theta^{A}\left(\nabla_{e_{B}} n\right)
$$

with $A, B=m+1, \ldots, 2 N$. Using the matrices $e_{\mu}^{A}$ and $e_{B}^{\nu}$ the shape operator can be expressed in terms of natural coordinates $x^{\mu}$ as

$$
\begin{aligned}
\mathscr{W}_{n}^{A}{ }_{B} & =-\theta^{A}\left(\nabla_{e_{B}} n\right)=-e_{\mu}^{A} \mathrm{e}_{B}^{\nu} \mathrm{d} x^{\mu}\left(\nabla_{\nu} n\right) \\
& =-e_{\mu}^{A} \mathrm{e}_{B}^{\nu} \mathrm{d} x^{\mu}\left[\left(\partial_{\nu} n^{\rho}+\Gamma_{\nu \lambda}^{\rho} n^{\lambda}\right) \partial_{\rho}\right]=-e_{\mu}^{A} \mathrm{e}_{B}^{\nu} \nabla_{\nu} n^{\mu} .
\end{aligned}
$$

The eigenvalues $k_{1}, \ldots, k_{2 N-m}$ of the matrix $\mathscr{W}_{n}$ are called principal curvatures. The average of the principal curvatures at a fixed point is called mean curvature $M_{n}$

$$
M_{n}=\frac{1}{2 N-m} \sum_{i=1}^{2 N-m} k_{i}=\frac{1}{2 N-m} \operatorname{Tr} \mathscr{W}_{n}=-\frac{1}{2 N-m} \sum_{A=3}^{2 N-m} g\left(\nabla_{e_{A}} n, e_{A}\right)
$$

Let us introduce the coarea formula (a generalization of the Fubini theorem) which allows expressing the integral of a function over the phase space $\Lambda$ in terms of integrals over the regular submanifolds of $\Lambda$. Let us introduce a coordinate system $\left\{u^{a}\right\}_{a=1, \ldots, 2 N}$ such that $u^{\bar{A}}=F^{\bar{A}}$ with $\bar{A}=1, \ldots, m$ on a region $\mathscr{M}_{\left[\mathrm{f}_{0}, \mathrm{f}_{1}\right]}=\left\{x \in \Lambda \mid f_{0}^{k} \leqslant F^{k}(x) \leqslant f_{1}^{k}\right\}$ free of critical points of function $f^{k}$. It follows that the metric $g$ reads

$$
g=g_{\mu \nu} \mathrm{d} x^{\mu} \otimes \mathrm{d} x^{\nu}=g_{a b} \mathrm{~d} u^{a} \otimes u^{b}=g_{\bar{A} \bar{B}} \mathrm{~d} u^{\bar{A}} \otimes \mathrm{d} u^{\bar{B}}+g_{A B} \mathrm{~d} u^{A} \otimes \mathrm{d} u^{B}
$$

where, if we consider the inverse matrices of the metric in the two coordinate systems we have 
Geometrical and topological study of the Kosterlitz-Thouless phase transition in the $\mathbf{X Y}$ model in two dimensions

$$
g^{\bar{A} \bar{B}}=g^{\mu \nu} \frac{\partial u^{\bar{A}}}{\partial x^{\mu}} \frac{\partial u^{\bar{B}}}{\partial x^{\nu}}=g\left(\nabla F^{\bar{A}}, \nabla F^{\bar{B}}\right) .
$$

The Riemannian volume element $\omega$ in the two coordinates systems reads

$$
\begin{aligned}
\omega & =\left|\operatorname{det} g_{\mu \nu}\right|^{1 / 2} \mathrm{~d} x^{1} \wedge \ldots \wedge \mathrm{d} x^{2 N}=\left|\operatorname{det} g_{a b}\right|^{1 / 2} \mathrm{~d} u^{1} \wedge \ldots \wedge \mathrm{d} u^{2 N}= \\
& =\left|\operatorname{det} g_{\bar{A} \bar{B}}\right|^{1 / 2} \mathrm{~d} u^{1} \wedge \ldots \wedge \mathrm{d} u^{m} \underbrace{\left|\operatorname{det} g_{A B}\right|^{1 / 2} \mathrm{~d} u^{m+1} \wedge \ldots \wedge \mathrm{d} u^{2 N}}_{\sigma_{\Sigma_{f 1}, \ldots, f m}}
\end{aligned}
$$

where $\sigma_{\Sigma_{f^{1}, \ldots, f m}}$ is the induced Riemannian area form on the submanifold $\Sigma_{f^{1}, \ldots, f^{m}}$. So any integral of a function $\psi\left(u^{\bar{A}}, u^{A}\right)$ on $\mathscr{M}_{\left[\mathbf{f}_{0}, \mathbf{f}_{1}\right]}$ can be expressed as

$$
\begin{aligned}
& \int_{\mathscr{M}_{\left[\mathrm{f}_{0}, \mathrm{f}_{1}\right]} \psi\left(u^{\bar{A}}, u^{A}\right) \omega=} \int_{\mathbf{f}_{0}}^{\mathbf{f}_{1}}\left(\int_{\Sigma_{u^{1}, \ldots, u^{m}}} \psi\left(u^{\bar{A}}, u^{A}\right)\left|\operatorname{det} g_{\bar{A} \bar{B}}\right|^{1 / 2} \sigma_{\Sigma_{u^{1}, \ldots, u^{m}}}\right) \\
& \times \mathrm{d} u^{1} \wedge \ldots \wedge \mathrm{d} u^{m}
\end{aligned}
$$

so it remains to evaluate $\left|\operatorname{det} g_{\bar{A} \bar{B}}\right|^{1 / 2}$. We start remembering that for any non-zero determinant matrix $\operatorname{det} A^{-1}=(\operatorname{det} A)^{-1}$, so it follows that

$$
\left|\operatorname{det} g_{\bar{A} \bar{B} \mid}\right|^{1 / 2}=\left|\operatorname{det} g^{\bar{A} \bar{B}}\right|^{-1 / 2}=\left[\mathbb{G}\left(\nabla F^{1}, \ldots, \nabla F^{m}\right)\right]^{-1 / 2}
$$

where $\mathbb{G}\left(X^{1}, \ldots, X^{m}\right):=\operatorname{det}\left[g\left(X^{i}, X^{j}\right)_{i, j=1, \ldots, m}\right]$ is the so called Grammian, so that the expression (45) takes the final form

$$
\begin{aligned}
\int_{M_{\left[\mathfrak{f}_{0}, \mathbf{f}_{1}\right]} \psi\left(u^{A}, u^{\bar{A}}\right) \omega=} & \int_{\mathbf{f}_{0}}^{\mathbf{f}_{1}}\left(\int_{\Sigma_{u^{1}, \ldots, u^{m}}} \psi\left(u^{A}, u^{\bar{A}}\right) \frac{\sigma_{\Sigma_{u^{1}, \ldots, u^{m}}}}{\sqrt{\mathbb{G}\left(\nabla F^{1}, \ldots, \nabla F^{m}\right)}}\right) \\
& \times \mathrm{d} u^{1} \wedge \ldots \wedge \mathrm{d} u^{m},
\end{aligned}
$$

that is usually referred to as coarea formula. When $\psi\left(u^{\bar{A}}, u^{A}\right)=\prod_{\bar{A}=1}^{m} \delta\left(u^{\bar{A}}-f^{\bar{A}}\right)$, then the equation (47) can be interpreted as the microcanonical partition function $\Omega_{N}\left(f^{1}, \ldots, f^{m}\right)$ where $m$ independent first integral of motion $\left\{F^{\bar{A}}\right\}_{A=1, \ldots, m}$ has been fixed, i.e.

$$
\Omega_{N}\left(f^{1}, \ldots, f^{m}\right)=\int_{\Sigma_{f^{1}, \ldots, f^{m}}} \frac{\sigma_{\Sigma_{f^{1}, \ldots, f^{m}}}}{\sqrt{\mathbb{G}\left(\nabla F^{1}, \ldots, \nabla F^{m}\right)}}=\int_{\Sigma_{f^{1}, \ldots, f^{m}}} \sigma_{f^{1}, \ldots, f^{m}}
$$

\section{A.2. Differential geometrical structure of co-dimension two submanifold $\Sigma_{E, \mathscr{P}}$ in phase space}

In this section the mathematical tools outlined in the previous section, for codimension$m$ submanifolds $\Sigma_{f^{1}, \ldots, f^{m}}$, are used to study the $X Y$-2D model. In what follows, the bridge between thermodynamics and geometry of the regular submanifolds $\Sigma_{E, \mathscr{P}}$ of phase space $\Sigma_{E, \mathscr{P}}=\{x \in \Lambda \mid H(x)=E \wedge P(x)=p\}$ is given in the framework of a (generalized) 
Geometrical and topological study of the Kosterlitz-Thouless phase transition in the $\mathbf{X Y}$ model in two dimensions microcanonical ensemble where both the total energy

$$
H=\frac{1}{2} \sum_{\tilde{\mu}=1}^{N} p^{\tilde{\mu}} p_{\tilde{\mu}}+V(q)
$$

and the total momentum

$$
P=\sum_{\tilde{\mu}=1}^{N} p^{\tilde{\mu}}
$$

are conserved quantities.

The first step to study the extrinsic geometry of these manifolds consists in defining the normal bundle of $\Sigma_{E, \mathscr{P}}$. This requires to compute both the gradient of the total momentum

$$
\nabla P=\sum_{\tilde{\mu}} \partial_{\tilde{\mu}} \Longrightarrow\|\nabla P\|=N^{1 / 2}
$$

and the gradient of the Hamiltonian $H$

$$
\nabla H=\sum_{\tilde{\mu}} p^{\tilde{\mu}} \partial_{\tilde{\mu}}+\sum_{\hat{\mu}} \partial^{\hat{\mu}} V \partial_{\hat{\mu}} \Longrightarrow\|\nabla H\|=\left[\sum_{\tilde{\mu}} p^{\tilde{\mu}} p_{\tilde{\mu}}+\|\hat{\nabla} V\|^{2}\right]^{1 / 2},
$$

where $\hat{\nabla} f=\partial^{\hat{\mu}} f \partial_{\hat{\mu}}$ is the gradient referred only to the set of generalized coordinates $q^{\hat{\mu}}$.

Two normal vector fields can be associated to the two gradient fields in the absence of critical points

$$
\begin{aligned}
& n_{P}=\frac{\nabla P}{\|\nabla P\|}=\frac{\sum_{\tilde{\mu}} \partial_{\tilde{\mu}}}{N^{1 / 2}} \\
& n_{H}=\frac{\nabla H}{\|\nabla H\|}=\frac{\sum_{\tilde{\mu}} p^{\tilde{\mu}} \partial_{\tilde{\mu}}+\sum_{\hat{\mu}} \partial^{\hat{\mu}} V \partial_{\hat{\mu}}}{\left[\sum_{\tilde{\mu}} p^{\tilde{\mu}} p_{\tilde{\mu}}+\|\hat{\nabla} V\|^{2}\right]^{1 / 2}}
\end{aligned}
$$

Following the definition of the microcanonical volume $\Omega_{N}$ given in appendix A.1, equation (48) reads in this case

$$
\begin{aligned}
\Omega_{N}(E, \mathscr{P}) & =\int_{\Lambda} \delta(H(p, q)-E) \delta(P-\mathscr{P}) \omega=\int_{\Sigma_{E, \mathscr{P}}} \frac{\sigma_{\Sigma_{E, \mathscr{P}}}}{\sqrt{\mathbb{G}(\nabla H, \nabla P)}} \\
& =\int_{\Sigma_{E, \mathscr{P}}} \chi \sigma_{\Sigma_{E, \mathscr{P}}}=\int_{\Sigma_{E, \mathscr{P}}} \sigma_{E, \mathscr{P}},
\end{aligned}
$$

where the Riemannian volume form on the manifold $\Lambda$ is $\omega=\sqrt{\operatorname{det} g} \mathrm{~d} p^{1} \wedge \mathrm{d} q^{1} \ldots \wedge$ $\mathrm{d} p^{N} \wedge \mathrm{d} q^{N}$, with $g$ the assigned metric structure over $\Lambda$, and the induced form of 
Geometrical and topological study of the Kosterlitz-Thouless phase transition in the $\mathbf{X Y}$ model in two dimensions

Riemannian area on the submanifold $\Sigma_{E, \mathscr{P}}$ is $\sigma_{\Sigma_{E, \mathscr{P}}}$, and

$$
\begin{aligned}
\chi & =(\sqrt{\mathbb{G}(\nabla H, \nabla P)})^{-1} \\
& =\left(\sqrt{\|\nabla H\|^{2}\|\nabla P\|^{2}-(g(\nabla H, \nabla P))^{2}}\right)^{-1}
\end{aligned}
$$

where $\mathbb{G}\left(X^{1}, \ldots, X^{m}\right):=\operatorname{det}\left[g\left(X^{i}, X^{j}\right)_{i, j=1, \ldots, m}\right]$ is the Grammian. Finally, to simplify the notation, we denoted with $\chi \sigma_{\Sigma_{E, \mathscr{P}}}=\sigma_{E, \mathscr{P}}$.

As in what follows, we will be interested in the derivative with respect to $E$ at fixed $\mathscr{P}$, let us introduce the vector field $\xi$, which has to fulfil the two following conditions:

$$
\begin{aligned}
& \mathrm{d} H(\xi)=1 \\
& \mathrm{~d} P(\xi)=0
\end{aligned}
$$

and using the gradient definition (34), we obtain

$$
\begin{aligned}
& g(\nabla H, \xi)=1 \\
& g(\nabla P, \xi)=0 .
\end{aligned}
$$

After further calculations, it is found that

$$
\xi=c\left(\nabla H-g(\nabla H, \nabla P) \frac{\nabla P}{\|\nabla P\|^{2}}\right),
$$

with $c=\left(\|\nabla H\|^{2}-\frac{g(\nabla H, \nabla P)^{2}}{\|\nabla P\|^{2}}\right)^{-1}$.

We are interested in studying the extrinsic geometry of the codimension-two submanifolds of phase space $\Sigma_{E, \mathscr{P}}$ when $\mathscr{P}$ is fixed while $E$ can change. So, we simplify the problem by considering $\Sigma_{E, \mathscr{P}}$ as a codimension-one submanifold, immersed into $\Sigma_{\mathscr{P}}$, foliated by the level sets $H=E$. Thus we introduce the projector operator $\mathbb{P}_{\Sigma_{E . \mathscr{P}}}$ for vectors on the tangent bundle $T \Sigma_{\mathscr{P}}$, i.e.

$$
\mathbb{P}_{\Sigma_{E, \mathscr{P}}}(X)=X-g\left(X, n_{P}\right) n_{P}
$$

Let us redefine the two normal vector fields on $\Sigma_{E, \mathscr{P}}$

$$
\begin{aligned}
& n_{1}=n_{P} \\
& n_{2}=\frac{\mathbb{P}_{\Sigma_{E, \mathscr{P}}}\left(n_{H}\right)}{\left\|\mathbb{P}_{\Sigma_{E, \mathscr{P}}}\left(n_{H}\right)\right\|}=\frac{\mathbb{P}_{\Sigma_{E, \mathscr{P}}}(\nabla H)}{\left\|\mathbb{P}_{\Sigma_{E, \mathscr{P}}}(\nabla H)\right\|},
\end{aligned}
$$

where $n_{2}$ is the normal vector field to the energy level sets on $\Sigma_{\mathscr{P}}$. We notice that

$$
\begin{aligned}
\mathbb{P}_{\Sigma_{E, \mathscr{P}}}(\nabla H) & =\nabla H-g\left(\nabla H, \frac{\nabla P}{\|\nabla P\|}\right) \frac{\nabla P}{\|\nabla P\|}=\nabla H-\frac{1}{N} g(\nabla H, \nabla P) \nabla P \\
& =\nabla H-\frac{1}{N} P \nabla P=\nabla H-\frac{1}{N} \nabla\left(\frac{P^{2}}{2}\right)=\nabla f_{\mathrm{HP}},
\end{aligned}
$$


Geometrical and topological study of the Kosterlitz-Thouless phase transition in the $\mathbf{X Y}$ model in two dimensions where we have introduced the function

$$
f_{\mathrm{HP}}=H-\frac{1}{N} \frac{P^{2}}{2} \text {. }
$$

With the definition of the function $f_{\mathrm{HP}}$, the vector field $\xi$ introduced in equation (58) can be more easily expressed as follows

$$
\xi=\frac{\nabla f_{\mathrm{HP}}}{\left\|\nabla f_{\mathrm{HP}}\right\|^{2}}
$$

It is natural to introduce the differential forms $\theta^{1}=e_{\mu}^{1} \mathrm{~d} x^{\mu}$ and $\theta^{2}=e_{\mu}^{2} \mathrm{~d} x^{\mu}$, that, in terms of derivatives of $f_{\mathrm{HP}}$ and $P$, read

$$
\begin{aligned}
& \imath_{n_{1}} \theta^{1}=e_{\mu}^{1} n_{1}^{\mu}=e_{\mu}^{1} \frac{\partial^{\mu} P}{\|\nabla P\|}=1 \quad \Rightarrow \quad e_{\mu}^{1}=\frac{\partial_{\mu} P}{\|\nabla P\|} \\
& \imath_{n_{2}} \theta^{2}=e_{\mu}^{2} n_{2}^{\mu}=e_{\mu}^{2} \frac{\partial^{\mu} f_{\mathrm{HP}}}{\left\|\nabla f_{\mathrm{HP}}\right\|}=1 \quad \Rightarrow \quad e_{\mu}^{2}=\frac{\partial_{\mu} f_{\mathrm{HP}}}{\left\|\nabla f_{\mathrm{HP}}\right\|}
\end{aligned}
$$

whence

$$
\begin{aligned}
& \theta^{1}=\frac{\partial_{\mu} P \mathrm{~d} x^{\mu}}{\|\nabla P\|}=\frac{\mathrm{d} P}{\|\nabla P\|} \\
& \theta^{2}=\frac{\partial_{\mu} f_{\mathrm{HP}} \mathrm{d} x^{\mu}}{\left\|\nabla f_{\mathrm{HP}}\right\|}=\frac{\mathrm{d} f_{\mathrm{HP}}}{\left\|\nabla f_{\mathrm{HP}}\right\|} .
\end{aligned}
$$

With this notation, the volume form on the phase space can be rewritten as follows

$$
\omega=\theta^{1} \wedge \theta^{2} \wedge \underbrace{\theta^{2+1} \wedge \ldots \wedge \theta^{2 N}}_{\sigma_{\Sigma_{E, \mathscr{P}}}}=\theta^{1} \wedge \theta^{2} \wedge \sigma_{\Sigma_{E, \mathscr{P}}}
$$

where $\sigma_{\Sigma_{E, \mathscr{P}}}$ is the induced Riemannian area form on the submanifold $\Sigma_{E, \mathscr{P}}$.

\section{A.3. Federer-Lawrence formula for co-dimension two manifold and microcanonical thermodynamic observables}

Since the relevant thermodynamic observables are given by derivatives of thermodynamic potentials (which depend on the statistical ensemble adopted), it is useful to provide a derivation formula for the integral of functions over $\Sigma_{E, \mathscr{P}}$ with respect to the energy at fixed total momentum $\mathscr{P}$.

Let us define the function $\mathscr{H}:\left(E_{1}, E_{2}\right) \rightarrow \mathbb{R}$, related to a generic regular function $h \in C^{\infty}(\Lambda)$, as

$$
\mathscr{H}(E, \mathscr{P})=\int_{\Sigma_{E, \mathscr{P}}} h \sigma_{E, \mathscr{P}} .
$$

Then we derive the explicit form of the operator $\mathscr{A}_{E}(\cdot)$ acting on $h$ s.t.

$$
\frac{\partial \mathscr{H}}{\partial E}(E, \mathscr{P})=\int_{\Sigma_{E, \mathscr{P}}} \mathscr{A}_{E}(h) \sigma_{E, \mathscr{P}} .
$$


Geometrical and topological study of the Kosterlitz-Thouless phase transition in the $\mathbf{X Y}$ model in two dimensions

To work out $\mathscr{A}_{E}(\cdot)$, using the notation $\mathscr{H}^{\prime}(E, \mathscr{P})=\partial_{E} \mathscr{H}(E, \mathscr{P})$ and $\Omega^{\prime}(E)=$ $\partial_{E} \Omega(E, \mathscr{P})$, consider

$$
\begin{aligned}
\mathscr{H}^{\prime}(E, \mathscr{P}) & =\lim _{\Delta E \rightarrow 0} \frac{\int_{\Sigma_{\mathscr{P}, E}+\Delta E} h \sigma_{\mathscr{P}, E}-\int_{\Sigma_{\mathscr{P}, E}} h \sigma_{\mathscr{P}, E}}{\Delta E} \\
& =\lim _{\Delta E \rightarrow 0} \frac{\int_{\phi\left(\Sigma_{\mathscr{P}, E}, \Delta E\right)} h \sigma_{\mathscr{P}, E}-\int_{\Sigma_{\mathscr{P}, E}} h \sigma_{\mathscr{P}, E}}{\Delta E} \\
& =\lim _{\Delta E \rightarrow 0} \frac{\int_{\Sigma_{\mathscr{P}, E}} \phi_{\Delta E}^{*}\left(h \sigma_{\mathscr{P}, E}\right)-\int_{\Sigma_{\mathscr{P}, E}} h \sigma_{\mathscr{P}, E}}{\Delta E} \\
& =\int_{\Sigma_{\mathscr{P}, E}}\left[\lim _{\Delta E \rightarrow 0} \frac{\phi_{\Delta E}^{*}\left(h \sigma_{\mathscr{P}, E}\right)-h \sigma_{\mathscr{P}, E}}{\Delta E}\right] \\
& =\int_{\Sigma_{\mathscr{P}, E}} \mathscr{L}_{\xi}\left(h \sigma_{\mathscr{P}, E}\right) .
\end{aligned}
$$

On the third line $\phi_{\Delta E}^{*}$ is the pullback of the one-parameter group of diffeomorphisms $\phi(\cdot, \Delta E)$ among the energy level sets generated by a vector field $\xi$ defined below. In the last term, the integral is the Lie derivative along the vector field $\xi$ of the $(2 N-2)$-form $\alpha=h \sigma_{\mathscr{P}, E}=h \chi \sigma_{\Sigma_{\mathscr{P}, E}}$, so we obtain

$$
\mathscr{L}_{\xi}\left(h \sigma_{E, \mathscr{P}}\right)=\xi(h \chi) \sigma_{\Sigma_{E, \mathscr{P}}}+h \chi \mathscr{L}_{\xi}\left(\sigma_{\Sigma_{E, \mathscr{P}}}\right) .
$$

In order to evaluate the Lie derivative in equation (69) of the induced Riemannian measure $\sigma_{\Sigma_{E, \mathscr{P}}}$ we use the homotopy formula

$$
\begin{aligned}
\mathscr{L}_{\xi}\left(\sigma_{\Sigma_{E, \mathscr{P}}}\right) & =\left[\imath_{\xi} \mathrm{d}+\mathrm{d} \imath_{\xi}\right] \sigma_{\Sigma_{E, \mathscr{P}}}=\imath_{\xi}\left(\mathrm{d} \imath_{n_{2}} \imath_{n_{1}}\right) \omega=\left\|\nabla f_{H_{P}}\right\|^{-1} \imath_{n_{2}}\left(\mathrm{~d} \imath_{n_{2}} \imath_{n_{1}}\right) \omega \\
& =\left\|\nabla f_{H_{P}}\right\|^{-1} \imath_{n_{2}} \mathrm{~d}\left(\theta^{3} \wedge \ldots \wedge \theta^{2 N}\right) \\
& =\left\|\nabla f_{\mathrm{HP}}\right\|^{-1} \operatorname{div}\left(n_{2}\right) \sigma_{\Sigma_{E, \mathscr{P}}},
\end{aligned}
$$

that is substituted in equation (69)

$$
\mathscr{L}_{\xi}\left(h \sigma_{E, \mathscr{P}}\right)=\left(\frac{\mathscr{L}_{\xi}(h \chi)}{\chi}+h\left\|\nabla f_{\mathrm{HP}}\right\|^{-1} \operatorname{div}\left(n_{2}\right)\right) \chi \sigma_{\Sigma_{E, \mathscr{P}}}=\mathscr{A}_{E}(h) \sigma_{E, \mathscr{P}} .
$$

So we have obtained that

$$
\mathscr{H}^{\prime}(E, \mathscr{P})=\int_{\Sigma_{E, \mathscr{P}}} \mathscr{A}_{E}(h) \sigma_{E, \mathscr{P}},
$$

that generalizes to higher derivatives

$$
\mathscr{H}^{(k)}(E, \mathscr{P})=\int_{\Sigma_{E, \mathscr{P}}} \mathscr{A}_{E}^{k}(h) \sigma_{E, \mathscr{P}}=\int_{\Sigma_{E, \mathscr{P}}} \underbrace{\mathscr{A}_{E}\left(\mathscr{A}_{E}\left(\ldots\left(\mathscr{A}_{E}(h)\right)\right)\right)}_{k \text {-times }} \sigma_{E, \mathscr{P}} .
$$

By simply putting $h=1$, one obtains

$$
\Omega_{N}^{\prime}(E)=\int_{\Sigma_{E, \mathscr{P}}}\left(\frac{\mathscr{L}_{\xi}(\chi)}{\chi}+\frac{\operatorname{div}\left(n_{2}\right)}{\left\|\nabla f_{\mathrm{HP}}\right\|}\right) \chi \sigma_{\Sigma_{E, \mathscr{P}}},
$$



with

$$
\begin{aligned}
\mathscr{L}_{\xi}(\chi)=\nabla_{\xi}(\chi) & =\frac{1}{\left\|\nabla f_{\mathrm{HP}}\right\|^{2}} \nabla_{\nabla_{f_{\mathrm{HP}}}}\left(\frac{1}{\left\|\nabla f_{\mathrm{HP}}\right\| N^{1 / 2}}\right) \\
& =\left(-\frac{1}{2 N^{1 / 2}}\right) \frac{1}{\left\|\nabla f_{\mathrm{HP}}\right\|^{2}} \frac{2 g\left(\nabla f_{H P}, \nabla_{\nabla_{f_{\mathrm{HP}}}} \nabla f_{\mathrm{HP}}\right)}{\left\|\nabla f_{\mathrm{HP}}\right\|^{3}} \\
& =-\frac{\operatorname{Hess} f_{\mathrm{HP}}\left(\nabla f_{\mathrm{HP}}, \nabla f_{\mathrm{HP}}\right)}{\left\|\nabla f_{\mathrm{HP}}\right\|^{5} N^{1 / 2}},
\end{aligned}
$$

where we have used the definition of the Hessian two-covector

$$
\operatorname{Hess} f(X, Y) \equiv g\left(\nabla_{X} \nabla f, Y\right)=X \mathrm{~d} f(Y)-\mathrm{d} f\left(\nabla_{X} Y\right) .
$$

So we have

$$
\Omega_{N}^{\prime}(E)=\int_{\Sigma_{E, \mathscr{P}}}\left(-\frac{\operatorname{Hess} f_{\mathrm{HP}}\left(\nabla_{f_{\mathrm{HP}}}, \nabla_{f_{\mathrm{HP}}}\right)}{\left\|\nabla f_{\mathrm{HP}}\right\|^{4}}+\frac{\operatorname{div}\left(n_{2}\right)}{\left\|\nabla f_{\mathrm{HP}}\right\|}\right) \sigma_{E, \mathscr{P}} .
$$

Equation (74) can be writen also in this form

$$
\begin{aligned}
\Omega_{N}^{\prime}(E) & =\int_{\Sigma_{E, \mathscr{P}}}\left(\mathscr{L}_{n_{2}}(\|\xi\|)+\|\xi\| \operatorname{div}\left(n_{2}\right)\right) \chi \sigma_{\Sigma_{E, \mathscr{P}}} \\
& =\int_{\Sigma_{E, \mathscr{P}}} \operatorname{div}\left(n_{2}\|\xi\|\right) \sigma_{E, \mathscr{P}} \\
& =\int_{\Sigma_{E, \mathscr{P}}} \operatorname{div}(\xi) \sigma_{E, \mathscr{P}} .
\end{aligned}
$$

It follows that the inverse of the microcanonical geometrical temperature is given by

$$
T_{\text {geo }}^{-1}(E, \mathscr{P})=\frac{\int_{\Sigma_{E, \mathscr{P}}} \operatorname{div} \xi \sigma_{E, \mathscr{P}}}{\int_{\Sigma_{E, \mathscr{P}}} \sigma_{E, \mathscr{P}}}=\int_{\Sigma_{E, \mathscr{P}}} \mu \operatorname{div} \xi=\langle\operatorname{div} \xi\rangle_{\mu c}(E, \mathscr{P}),
$$

where $\langle\cdot\rangle_{\mu c}(E, \mathscr{P})$ indicates the averages over the energy level sets $\Sigma_{E, \mathscr{P}}$ with the probability measure $\mu$.

\section{A.4. Geometrical and topological observables in $X Y$ 2D model}

As we are interested in the extrinsic geometry of $\Sigma_{E, \mathscr{P}}$, the mean curvature along the vector $n_{2}$ is introduced to give, according to equation (41), 
Geometrical and topological study of the Kosterlitz-Thouless phase transition in the $\mathbf{X Y}$ model in two dimensions

$$
\begin{aligned}
M_{n_{2}} & =-\frac{1}{2 N-2} \sum_{A=3}^{2 N-2} g\left(\nabla_{e_{A}} n_{2}, e_{A}\right) \\
& =-\frac{1}{2 N-2}\left[\sum_{i=1}^{2} g\left(\nabla_{n_{i}} n_{2}, n_{i}\right)+\sum_{A=3}^{2 N-2} g\left(\nabla_{e_{A}} n_{2}, e_{A}\right)-\sum_{i=1}^{2} g\left(\nabla_{n_{i}} n_{2}, n_{i}\right)\right] \\
& =-\frac{1}{2 N-2}\left[\sum_{\tilde{\mu}} g\left(\nabla_{\partial_{\tilde{\mu}}} n_{2}, \partial_{\tilde{\mu}}\right)+\sum_{\hat{\mu}} g\left(\nabla_{\partial_{\hat{\mu}}} n_{2}, \partial_{\hat{\mu}}\right)-g\left(\nabla_{n_{1}} n_{2}, n_{1}\right)-g\left(\nabla_{n_{2}} n_{2}, n_{2}\right)\right] \\
& =-\frac{1}{2 N-2}\left[\operatorname{div}\left(n_{2}\right)-g\left(\nabla_{n_{1}} n_{2}, n_{1}\right)\right],
\end{aligned}
$$

where the vector fields $e_{A}$ belong to the tangent bundle of the submanifold $\Sigma_{E, \mathscr{P}}$, i.e. $e_{A} \in T \Sigma_{E, \mathscr{P}}$.

The divergence of the vector field $n_{2}$ can be rewritten as

$$
\begin{aligned}
& \operatorname{div}\left(n_{2}\right)=\operatorname{div}\left(\frac{\nabla f_{\mathrm{HP}}}{\left\|\nabla f_{\mathrm{HP}}\right\|}\right)=\frac{\operatorname{div}\left(\nabla f_{\mathrm{HP}}\right)}{\left\|\nabla f_{\mathrm{HP}}\right\|}-\frac{1}{2} \frac{\nabla \nabla f_{\mathrm{HP}}\left\|\nabla f_{\mathrm{HP}}\right\|^{2}}{\left\|\nabla f_{\mathrm{HP}}\right\|^{3}}= \\
& =\frac{\Delta f_{\mathrm{HP}}}{\left\|\nabla f_{\mathrm{HP}}\right\|}-\frac{g\left(\nabla f_{\mathrm{HP}}, \nabla_{\nabla_{f_{\mathrm{HP}}}} \nabla f_{\mathrm{HP}}\right)}{\left\|\nabla f_{\mathrm{HP}}\right\|^{3}} \\
& =\frac{\Delta f_{\mathrm{HP}}}{\left\|\nabla f_{\mathrm{HP}}\right\|}-\frac{\operatorname{Hess} f_{\mathrm{HP}}\left(\nabla f_{\mathrm{HP}}, \nabla f_{\mathrm{HP}}\right)}{\left\|\nabla f_{\mathrm{HP}}\right\|^{3}}
\end{aligned}
$$

The second term of the last equation in (80) is null. In fact, from $\nabla_{\nabla P} \nabla f_{\mathrm{HP}}=0$ it follows

$$
\begin{aligned}
\nabla_{n_{1}} n_{2}= & \frac{1}{\|\nabla P\|} \nabla_{\nabla P}\left(\frac{\nabla f_{\mathrm{HP}}}{\left\|\nabla f_{\mathrm{HP}}\right\|}\right)=\frac{\nabla_{\nabla P} \nabla f_{\mathrm{HP}}}{\|\nabla P\|\left\|\nabla f_{\mathrm{HP}}\right\|} \\
& -\frac{\left(\nabla_{\nabla P} \nabla f_{\mathrm{HP}}, \nabla f_{\mathrm{HP}}\right) \nabla f_{\mathrm{HP}}}{\|\nabla P\|\left\|\nabla f_{\mathrm{HP}}\right\|^{3}}=0 .
\end{aligned}
$$

Finally the mean curvature $M_{n_{2}}$ of $\Sigma_{E, \mathscr{P}}$ reads

$$
M_{n_{2}}=\frac{1}{2 N-2}\left[\frac{\operatorname{Hess} f_{\mathrm{HP}}\left(\nabla f_{\mathrm{HP}}, \nabla f_{\mathrm{HP}}\right)}{\left\|\nabla f_{\mathrm{HP}}\right\|^{3}}-\frac{\Delta f_{\mathrm{HP}}}{\left\|\nabla f_{\mathrm{HP}}\right\|}\right] .
$$

To get some meaningful information about the topology of the co-dimension-one manifolds $\Sigma_{E, \mathscr{P}}$ immersed in the euclidean space $\Sigma_{\mathscr{P}}$, the dispersion of the principal curvatures $\sigma_{n_{2}}^{2}\left(k_{i}\right)$ is computed from

$$
\sigma_{n 2}^{2}\left(k_{i}\right)=\left\langle k_{i}^{2}\right\rangle-\left\langle k_{i}\right\rangle^{2}=\frac{\operatorname{Tr}\left(\mathscr{W}_{n_{2}}^{2}\right)}{2 N-2}-\frac{\left(\operatorname{Tr} \mathscr{W}_{n_{2}}\right)^{2}}{(2 N-2)^{2}},
$$


Geometrical and topological study of the Kosterlitz-Thouless phase transition in the $\mathbf{X Y}$ model in two dimensions where $\mathscr{W}$ is the shape-operator (Weingarten map) defined in equation (37), and

$$
\begin{aligned}
\operatorname{Tr}\left(\mathscr{W}_{n_{2}}^{2}\right) & =\sum_{A=3}^{2 N-2} g\left(-\nabla_{\left(-\nabla_{e_{A}} n_{2}\right)} n_{2}, e_{A}\right)=-\sum_{A=3}^{2 N-2} g\left(n_{2}, \nabla_{\nabla_{e_{A}} n_{2}} e_{A}\right) \\
& =-\left[\sum_{A=3}^{2 N-2} g\left(n_{2}, \nabla_{e_{A}} \nabla_{e_{A}} n_{2}\right)+g\left(n_{2},\left[\nabla_{e_{A}} n_{2}, e_{A}\right]\right)\right] \\
& =\sum_{A=3}^{2 N-2}\left[g\left(\nabla_{e_{A}} n_{2}, \nabla_{e_{A}} n_{2}\right)+g\left(n_{2},\left[e_{A}, \nabla_{e_{A}} n_{2}\right]\right)\right] \\
& =\sum_{\mu=1}^{2 N} g\left(\nabla_{\mu} n_{2}, \nabla_{\mu} n_{2}\right)+\sum_{A=3}^{2 N-2} g\left(n_{2},\left[e_{A}, \nabla_{e_{A}} n_{2}\right]\right)-g\left(\nabla_{n_{2}} n_{2}, \nabla_{n_{2}} n_{2}\right) \\
& =\sum_{\mu=1}^{2 N}\left\|\nabla_{\mu} n_{2}\right\|^{2}-\left\|\nabla_{n_{2}} n_{2}\right\|^{2}+\sum_{A=3}^{2 N-2} g\left(n_{2},\left[e_{A}, \nabla_{e_{A}} n_{2}\right]\right) .
\end{aligned}
$$

The first term of the equation (85) is given by

$$
\begin{aligned}
\sum_{\mu=1}^{2 N}\left\|\nabla_{\mu} n_{2}\right\|^{2}= & \sum_{\mu=1}^{2 N}\left[\frac{\partial_{\mu} \partial^{\rho} f_{\mathrm{HP}} \partial_{\rho} \partial_{\gamma} f_{\mathrm{HP}}}{\left\|\nabla f_{\mathrm{HP}}\right\|^{2}}+\frac{\partial^{\rho} f_{\mathrm{HP}} \partial_{\rho} f_{\mathrm{HP}}}{\left\|\nabla f_{\mathrm{HP}}\right\|^{6}}\right. \\
& \times\left(\partial_{\mu} \partial_{\nu} f_{\mathrm{HP}} \partial^{\nu} f_{\mathrm{HP}}\right)\left(\partial_{\gamma} \partial_{\sigma} f_{\mathrm{HP}} \partial^{\sigma} f_{\mathrm{HP}}\right)+ \\
& \left.-2 \frac{\left(\partial_{\mu} \partial^{\rho} f_{\mathrm{HP}} \partial_{\rho} f_{\mathrm{HP}}\right)\left(\partial_{\gamma} \partial_{\nu} f_{\mathrm{HP}} \partial^{\nu} f_{\mathrm{HP}}\right.}{\left\|\nabla f_{\mathrm{HP}}\right\|^{4}}\right] \delta_{\mu}^{\gamma} \\
= & \sum_{\mu=1}^{2 N}\left[\frac{\partial_{\mu} \partial^{\rho} f_{\mathrm{HP}} \partial_{\rho} \partial_{\gamma} f_{\mathrm{HP}}}{\left\|\nabla f_{\mathrm{HP}}\right\|^{2}}-\frac{\left(\partial_{\mu} \partial^{\rho} f_{\mathrm{HP}} \partial_{\rho} f_{\mathrm{HP}}\right)\left(\partial_{\gamma} \partial_{\nu} f_{\mathrm{HP}} \partial^{\nu} f_{\mathrm{HP}}\right)}{\left\|\nabla f_{\mathrm{HP}}\right\|^{4}}\right] \delta_{\mu}^{\gamma} \\
= & \operatorname{Tr}\left[\frac{\left(\operatorname{Hess} f_{\mathrm{HP}}\right)^{2}}{\left\|\nabla f_{\mathrm{HP}}\right\|^{2}}\right]-\frac{\left(\operatorname{Hess} f_{\mathrm{HP}} \nabla f_{\mathrm{HP}}\right)^{2}}{\left\|\nabla f_{\mathrm{HP}}\right\|^{4}} .
\end{aligned}
$$

Knowing that

$$
\nabla_{n_{2}} n_{2}=\frac{\partial^{\mu} f_{\mathrm{HP}}}{\left\|\nabla f_{\mathrm{HP}}\right\|} \nabla_{\mu} n_{2}
$$

the second term then reads

$$
\begin{aligned}
\left\|\nabla_{n_{2}} n_{2}\right\|^{2} & =\frac{1}{\left\|\nabla f_{\mathrm{HP}}\right\|^{2}} g\left(\partial^{\mu} f_{\mathrm{HP}} \nabla_{\mu} n_{2}, \partial^{\sigma} f_{\mathrm{HP}} \nabla_{\sigma} n_{2}\right) \\
& =\frac{1}{\left\|\nabla f_{\mathrm{HP}}\right\|^{2}}\left[\frac{\left(\| \operatorname{Hess} f_{\mathrm{HP}}\right)\left(\nabla f_{\mathrm{HP}}\right) \|^{2}}{\left\|\nabla f_{\mathrm{HP}}\right\|^{2}}-\frac{\left\|\left(\operatorname{Hess} f_{H P}\right)\left(\nabla f_{\mathrm{HP}}\right)^{2}\right\|^{2}}{\left\|\nabla f_{\mathrm{HP}}\right\|^{4}}\right] .
\end{aligned}
$$


Geometrical and topological study of the Kosterlitz-Thouless phase transition in the $\mathbf{X Y}$ model in two dimensions

The last term of the equation (85) is a measure of the integrability of the vector field distribution $\left\{n_{1}, e_{\bar{A}}\right\}_{\bar{A}}$ in the sense of the Froebenius theorem, where $e_{\bar{A}} \in \mathrm{N} \Sigma_{E, \mathscr{P}}$, the normal bundle of the submanifold $\Sigma_{E, \mathscr{P}}$, with $\bar{A}=1,2$. In particular, if these fields form a closed algebra

$$
\left[n_{1}, e_{\bar{A}}\right] \in \operatorname{Span}\left\{n_{1}, e_{2+1}, \ldots, e_{2 N}\right\}
$$

then $\sum_{\bar{A}=3}^{2 N} g\left(n_{2},\left[\nabla_{e_{\bar{A}}} n_{2}, e_{\bar{A}}\right]\right)=0$.

It is convenient to rephrase this condition in the language of differential forms. Let us introduce the annihilator one-form $\eta$ defined as

$$
\begin{aligned}
\eta & =\left[\partial_{\tilde{\mu}} H-\left(\partial_{\tilde{\nu}} H \partial^{\tilde{\nu}} P\right) \partial_{\tilde{\mu}} P\right] \mathrm{d} q^{\tilde{\mu}}+\left[\partial_{\hat{\mu}} H-\left(\partial_{\hat{\nu}} H \partial^{\hat{\nu}} P\right) \partial_{\hat{\mu}} P\right] \mathrm{d} p^{\hat{\mu}} \\
& =\left[\partial_{\tilde{\mu}} H-\left(\partial_{\tilde{\nu}} H \partial^{\tilde{\nu}} P\right) \partial_{\tilde{\mu}} P\right] \mathrm{d} q^{\tilde{\mu}}+\partial_{\hat{\mu}} H \mathrm{~d} p^{\hat{\mu}}
\end{aligned}
$$

such that

$$
\eta\left(n_{1}\right)=\eta\left(e_{A}\right)=0 .
$$

So if $\mathrm{d} \eta$ is an annihilator two-form on the space $\operatorname{Span}\left\{n_{1}, e_{2+1}, \ldots, e_{N}\right\}$ then the fields $\left\{n_{1}, e_{A}\right\}_{A=2+1, \ldots, N}$ are in involution, i.e.

$$
\begin{aligned}
0=\mathrm{d} \eta(X, Y) & =X \eta(Y)-Y \eta(X)-\eta([X, Y]) \\
& =-\eta([X, Y]) \quad \forall X, Y \in \operatorname{Span}\left\{n_{1}, e_{1}, \ldots, e_{2 N-2}\right\} .
\end{aligned}
$$

For the one-form $\eta$ defined in (89) we can evaluate explicitly $\mathrm{d} \eta$,

$$
\begin{aligned}
\mathrm{d} \eta & =\left[\partial_{\tilde{\rho}} \partial_{\tilde{\mu}} H-\left(\partial_{\tilde{\rho}} \partial_{\tilde{\nu}} H \partial^{\tilde{\nu}} P+\partial_{\tilde{\nu}} H \partial_{\tilde{\rho}} \partial^{\tilde{\nu}} P\right) \partial_{\tilde{\mu}} P-\left(\partial_{\tilde{\nu}} H \partial^{\tilde{\nu}} P\right) \partial_{\tilde{\rho}} \partial_{\tilde{\mu}} P\right] \mathrm{d} q^{\tilde{\rho}} \wedge \mathrm{d} q^{\tilde{\mu}} \\
& +\partial_{\hat{\rho}} \partial_{\hat{\mu}} H \mathrm{~d} p^{\hat{\rho}} \wedge \mathrm{d} p^{\hat{\mu}} .
\end{aligned}
$$

The terms of the form $\partial_{\tilde{\rho}} \partial_{\tilde{\mu}} f$ and $\partial_{\hat{\rho}} \partial_{\hat{\mu}} f$ are symmetric in the permutation of the indexes and so are null. The derivative of order $k \geqslant 2$ of the function $P$ are null as it is a linear function in $p^{\tilde{\mu}}$. So the only nontrivial term is $\left(\partial_{\tilde{\rho}} \partial_{\tilde{\nu}} H \partial^{\tilde{\nu}} P\right) \partial_{\tilde{\mu}} P \mathrm{~d} p^{\tilde{\rho}} \wedge \mathrm{d} p^{\tilde{\mu}}$ : as $\left(\partial_{\tilde{\rho}} \partial_{\tilde{\nu}} H \partial^{\tilde{\nu}} P\right) \partial_{\tilde{\mu}} P=N$ it is not antisymmetric with respect to the indexes $\tilde{\rho}, \tilde{\mu}$ and the term is zero. So it follows that $\mathrm{d} \eta=0$. Finally

$$
\begin{aligned}
\operatorname{Tr}\left(\mathscr{W}_{n_{2}}^{2}\right)= & \operatorname{Tr}\left[\frac{\left(\operatorname{Hess} f_{\mathrm{HP}}\right)^{2}}{\left\|\nabla f_{\mathrm{HP}}\right\|^{2}}\right]-\frac{\left(\operatorname{Hess} f_{\mathrm{HP}} \nabla f_{\mathrm{HP}}\right)^{2}}{\left\|\nabla f_{\mathrm{HP}}\right\|^{4}}+ \\
& -\frac{1}{\left\|\nabla f_{\mathrm{HP}}\right\|^{2}}\left[\frac{\left(\| \operatorname{Hess} f_{\mathrm{HP}}\right)\left(\nabla f_{\mathrm{HP}}\right) \|^{2}}{\left\|\nabla f_{\mathrm{HP}}\right\|^{2}}-\frac{\left\|\left(\operatorname{Hess} f_{\mathrm{HP}}\right)\left(\nabla f_{\mathrm{HP}}\right)^{2}\right\|^{2}}{\left\|\nabla f_{\mathrm{HP}}\right\|^{4}}\right] .
\end{aligned}
$$

\section{References}

[1] Mermin N D and Wagner H 1966 Absence of ferromagnetism or antiferromagnetism in one- or two-dimensional isotropic Heisenberg models Phys. Rev. Lett. 171133

Mermin N D 1968 Crystalline order in two dimensions Phys. Rev. 176250 
[2] Elitzur S 1975 Impossibility of spontaneously breaking local symmetries Phys. Rev. D 123978

[3] Kosterlitz J M and Thouless D J 1973 Ordering, metastability and phase transitions in two-dimensional systems J. Phys. C: Solid State Phys. 61181

Kosterlitz J M 1974 The critical properties of the two-dimensional XY model J. Phys. C: Solid State Phys. 7 1046

[4] McBryan O A and Spencer T 1977 On the decay of correlations in $S O(n)$-symmetric ferromagnets Commun. Math. Phys. 53299

[5] Berezinskii V L 1972 Destruction of long-range order in one-dimensional and two-dimensional systems possessing a continuous symmetry group. II. Quantum systems Sov. Phys. JETP 34601

[6] Resnick D J, Garland J C, Boyd J T, Shoemaker S and Newrock R S 1981 Kosterlitz-Thouless transition in proximity-coupled superconducting arrays Phys. Rev. Lett. 471542

[7] Wu Z, Block J K and Bruun G M 2016 Liquid crystal phases of two-dimensional dipolar gases and Berezinskii-Kosterlitz-Thouless melting Sci. Rep. 619038

[8] Halperin B I and Nelson D R 1978 Theory of two-dimensional melting Phys. Rev. Lett. 41121

[9] Hadzibabic Z, Krüger P, Cheneau M, Battelier B and Dalibard J 2006 Berezinskii-Kosterlitz-Thouless crossover in a trapped atomic gas Nature 4411118

[10] Yang C N and Lee T D 1952 Statistical theory of equations of state and phase transitions. I. Theory of condensation Phys. Rev. 87 404-9

Lee T D and Yang C N 1952 Statistical theory of equations of state and phase transitions. II. Lattice gas and Ising model Phys. Rev. 87 410-9

[11] Pradzynski C C, Forck R M, Zeuch T, Slavicek P and Buck U 2012 A fully size-resolved perspective on the crystallization of water clusters Science 337 1529-32

[12] Leggett A J 2001 Bose-Einstein condensation in the alkali gases: some fundamental concepts Rev. Mod. Phys. 73 307-56 (erratum)

Leggett A J 2003 Bose-Einstein condensation in the alkali gases: some fundamental concepts Rev. Mod. Phys. 751083

[13] Lifshitz I M, Grosberg A Y and Khokhlov A R 1978 Some problems of the statistical physics of polymer chains with volume interaction Rev. Mod. Phys. 50683

[14] Dill K A, Banu Ozkan S, Shell M S and Weikl T R 2008 The protein folding problem Annu. Rev. Biophys. 37 289-316

[15] Georgii H O 2011 Gibbs Measures and Phase Transitions 2nd edn (Berlin: de Gruyter \& Co)

[16] Pettini M 2007 Geometry and Topology in Hamiltonian Dynamics and Statistical Mechanics (IAM Series No. 33) (Berlin: Springer)

[17] Franzosi R, Pettini M and Spinelli L 2007 Topology and phase transitions I. Preliminary results Nucl. Phys. B 782189

Franzosi R and Pettini M 2007 Topology and phase transitions II. Theorem on a necessary relation Nucl. Phys. B 782219

Kastner M and Mehta D 2011 Even though a counterexample was given in: Phase transitions detached from stationary points of the energy landscape Phys. Rev. Lett. 107160602

The problem can be fixed with a refinement of the hypotheses of the theorems, as shown in Gori M, Franzosi R and Pettini M 2018 Topological origin of phase transitions in the absence of critical points of the energy landscape J. Stat. Mech. 093204

[18] Pettini G, Gori M, Franzosi R, Clementi C and Pettini M 2019 On the origin of phase transitions in the absence of symmetry-breaking Phys. A 516 376-92

[19] Nerattini R, Kastner M, Mehta D and Casetti L 2013 Exploring the energy landscape of XY models Phys. Rev. E 87032140

[20] Kosterlitz J M 2017 Nobel lecture: topological defects and phase transitions Rev. Mod. Phys. 89040501

[21] Pinkall U 1986 Inequalities of Willmore type for submanifolds Math. Z. 193 241-6

[22] Nakahara M 1991 Geometry, Topology and Physics (Bristol: Adam Hilger)

It is worth remarking that Pinkall inequality becomes an equality if the Betti numbers $b_{\mathrm{i}}(\mathscr{M}), \mathrm{i}=0, \ldots, \mathrm{n}-\mathrm{m}$ are replaced by Morse numbers $\mu_{\mathrm{i}}(\mathscr{M}), \mathrm{i}=0, \ldots, \mathrm{n}-\mathrm{m}$, i.e. the number of critical points of a Morse function on $\mathscr{M}$ with index i. So the Pinkall inequality is a consequence of the so called weak Morse inequality, i.e. $\mu_{\mathrm{i}}(\mathscr{M}) \geqslant \mathrm{b}_{\mathrm{i}}(\mathscr{M})$

[23] Reed M and Simon B 1980 Functional analysis (Methods of Modern Mathematical Physics) vol 1 (New York: Academic)

[24] Cerruti-Sola M, Clementi C and Pettini M 2000 Hamiltonian dynamics and geometry of phase transitions in classical XY models Phys. Rev. E 615171 
[25] Schierz P, Zierenberg J and Janke W 2016 First-order phase transitions in the real microcanonical ensemble Phys. Rev. E 94 021301(R)

[26] Martin-Mayor V 2007 Microcanonical approach to the simulation of first-order phase transitions Phys. Rev. Lett. 98137207

[27] Casetti L 1995 Efficient symplectic algorithms for numerical simulations of Hamiltonian flows Phys. Scr. 5129

[28] Pettini M and Landolfi M 1990 Relaxation properties and ergodicity breaking in nonlinear Hamiltonian dynamics Phys. Rev. A 41768

[29] Bel-Hadj-Aissa G 2020 High order derivatives of Boltzmann microcanonical entropy with an additional conserved quantity Phys. Lett. A 384126449

[30] Pearson E M, Halicioglu T and Tiller W A 1985 Laplace-transform technique for deriving thermodynamic equations from the classical microcanonical ensemble Phys. Rev. A 323030

[31] Bachmann M 2014 Thermodynamics and Statistical Mechanics of Macromolecular Systems (Cambridge: Cambridge University Press)

[32] Qi K and Bachmann M 2018 Classification of phase transitions by microcanonical inflection-point Analysis Phys. Rev. Lett. 120180601

Bachmann M 2014 Thermodynamics and Statistical Mechanics of Macromolecular Systems (Cambridge: Cambridge University Press)

[33] Olsson P 1995 Monte Carlo analysis of the two-dimensional XY model. II. Comparison with the Kosterlitz renormalization-group equations Phys. Rev. B 524526

[34] Hasenbusch M 2005 The two-dimensional $X Y$ model at the transition temperature: a high-precision Monte Carlo study J. Phys. A: Math. Gen. 385869

[35] Tobochnik J and Chester G V 1979 Monte Carlo study of the planar spin model Phys. Rev. B 203761

[36] Gupta R, DeLapp J, Batrouni G G, Fox G C, Baillie C F and Apostolakis J 1988 Phase transition in the 2D XY model Phys. Rev. Lett. 611996

[37] Gupta R and Baillie C F 1992 Critical behavior of the two-dimensional XY model Phys. Rev. B 452883

[38] Machado T and Dupuis N 2010 From local to critical fluctuations in lattice models: a nonperturbative renormalization-group approach Phys. Rev. E 82041128

[39] Jakubczyk P and Eberlein A 2016 Thermodynamics of the two-dimensional $X Y$ model from functional renormalization Phys. Rev. E 93062145

[40] Franzosi R 2018 Microcanonical entropy for classical systems Phys. A 494 302-7

[41] The Betti numbers - as well as Morse indexes - are integers so that their sum, weighted or not, forms only staircase-like patterns which do not qualify as continuous and possibly differentiable functions. Actually the technical details of the reason why the corners of these staircase-like patterns are rounded can be found in section 9.5 of reference [16].

[42] Santos F A N, da Silva L C B and Coutinho-Filho M D 2017 Topological approach to microcanonical thermodynamics and phase transition of interacting classical spins J. Stat. Mech. 013202

[43] Cole A, Loges G J and Shiu G 2020 Quantitative and interpretable order parameters for phase transitions from persistent homology arXiv:2009.14231v1

[44] Donato I, Gori M, Pettini M, Petri G, De Nigris S, Franzosi R and Vaccarino F 2016 Persistent homology analysis of phase transitions Phys. Rev. E 93052138 